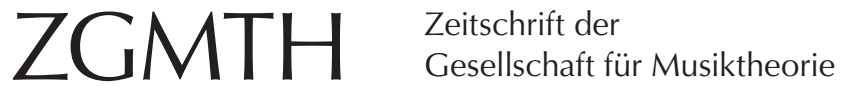

Poller, Tom Rojo (2017): The Interpretation is the Message. Komposition als angewandte Interpretation bei György Kurtág. ZGMTH 14/1, 93-131. https://doi.org/10.31751/889

(C) 2017 Tom Rojo Poller (trp@trpoller.de)

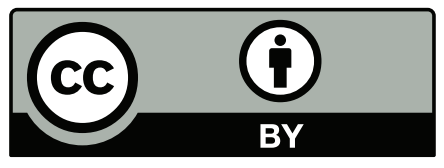

Dieser Text erscheint im Open Access und ist lizenziert unter einer Creative Commons Namensnennung 4.0 International Lizenz.

This is an open access article licensed under a Creative Commons Attribution 4.0 International License.

eingereicht / submitted: 31/08/2016

angenommen / accepted: 04/10/2016

veröffentlicht / first published: 30/06/2017

zuletzt geändert / last updated: 23/08/2018 


\title{
The Interpretation is the Message
}

\author{
Komposition als angewandte Interpretation bei György Kurtág
}

\author{
Tom Rojo Poller
}

\begin{abstract}
Als zutiefst in der Tradition europäischer Kunstmusik beheimatete scheint die Musik György Kurtágs auf den ersten Blick mit erprobten musikanalytischen Mitteln relativ gut erfassbar zu sein. Und tatsächlich lassen sich verschiedene Dimensionen von Kurtágs Musik anführen, die innerhalb etablierter Analysekategorien und -paradigmen plausibel beschrieben worden sind: die allgegenwärtigen Verweise und Anspielungen z. B. durch das Konzept von Intertextualität, die Grundlagen tonsetzerischen Handwerks durch historisch fundierte Strukturanalysen, semantische Gehalte durch hermeneutische Ansätze. Bislang wenig Beachtung allerdings fand ein im Kontext eines performance-theoretischen Interesses unmittelbar relevanter Aspekt, der für das musikalische Wirken des Komponisten ohne Frage von hoher Wichtigkeit ist: Interpretation. Ausgehend von Kurtágs Bemerkung, dass sein Komponieren wesentlich durch seine Auffassung von Interpretation motiviert sei, soll daher im vorliegenden Text der Vermutung nachgegangen werden, dass die Kategorie Interpretation in Kurtágs Werken eine wesentliche Rolle spielt und deswegen auch als Ausgangspunkt einer analytischen Erschließung dienen kann. Diese Arbeitshypothese wird in zwei methodischen Schritten verfolgt: Zunächst wird die Kategorie von Interpretation in Hinblick auf prominente Aspekte in Kurtágs Schaffen begrifflich aufgefächert. Im zweiten Schritt wird dann anhand von analytischen Betrachtungen zu Kurtágs Samuel Beckett: What is the Word op. 30a/b (1990/91) ansatzweise demonstriert, wie die so gewonnenen Einsichten für die rezeptive Interpretation einer konkreten Komposition fruchtbar gemacht werden könnten. Durch den Bezug auf drei in der Musikologie der letzten Jahrzehnte einflussreiche Paradigmen ([Inter-]Textualität, Medialität, Performativität) wird dabei eine explizit multiperspektivische Methodik gewählt, mithilfe derer darauf aufmerksam gemacht werden soll, dass eine palimpsestartige Überlagerung methodischer Perspektiven ein tiefendimensionales Verständnis von Musik zu befördern in der Lage ist.
\end{abstract}

At first sight, it seems fairly appropriate to apply standard music-analytical tools to the music of György Kurtág, since this music is deeply rooted in the tradition of European art music. Indeed, there are various dimensions of Kurtág's music which can be plausibly explained with the help of established analytical categories: the omnipresent references and allusions by the concept of intertextuality, the foundations of compositional craftsmanship by historically informed analyses of musical structures, semantic contents by hermeneutical approaches. However, little attention so far has been directed to an aspect that must be considered a crucial facet of Kurtág's work, being of special interest from the vantage point of musical performance studies: the aspect of interpretation. Taking its cue from Kurtág's remark that his composing is essentially motivated by his notion of interpretation, the present text assumes that the category of interpretation could 
be a key to open up new analytical perspectives on Kurtág's compositions. This hypothesis is pursued in two methodical steps. First, the category of interpretation is conceptually unfolded by dissecting prominent aspects of Kurtág's work which can be associated with a broad understanding of interpretation. In a second step, an analytical approach towards Kurtág's Samuel Beckett: What is the Word op. 30a/b (1990/91) is undertaken to demonstrate that such insights enrich the receptive interpretation of Kurtág's music. By employing three paradigms that have proven to be particularly influential in recent musicology ([inter-]textuality, mediality, performativity), the analysis aims at showing how the superposition of multiple perspectives can deepen the overall understanding of music.

»/Ch interessiere mich gar nicht für Konzerte mit meiner Musik. Vielleicht ist der eigentliche Grund für mein Komponieren der, meine Ideen von Interpretation zu verbreiten. " ${ }^{1}$

\section{METHODOLOGISCHE VORBEMERKUNG}

Während in weiten Teilen der kunst- und kulturwissenschaftlichen Disziplinen eine immer schneller und unüberblickbarer werdende Abfolge tatsächlich vollzogener oder auch nur proklamierter sturns` zu beobachten ist ${ }^{2}$, scheinen in Musikwissenschaft und Musiktheorie alleine die wirkmächtigsten dieser Paradigmenwechsel - und das zumeist deutlich zeitversetzt - nachvollzogen zu werden. Auf einer strukturellen Ebene mag das u.a. darauf zurückzuführen sein, dass die meisten Kunst- und Kulturwissenschaften - grob gesprochen - immer schon mit Bedeutungs- bzw. Sinnzusammenhängen ${ }^{3}$ umgehen, die selbst da, wo sie radikal infrage gestellt werden, immer noch als präfigurierte Grundlage des jeweiligen modus operandi dienen können (so etwa das Konzept sprachlicher Bedeutung in dekonstruktivistischen Ansätzen). Auf diese vorstrukturierten Sinnzusammenhänge können daher im Sinne des `Cross-Mapping-Prinzips ${ }^{4}$ immer wieder neue Orientierungsraster gelegt werden, um so neue Wege und damit unbeachtetes Terrain zu erschließen. Im Gegensatz dazu scheint die Musikologie wiederholt vor dem basalen Problem zu stehen, wie überhaupt die Grundelemente musikalischer Sinnkonstitution identifiziert und systematisch kartographiert werden können. Neben einer gewissen methodischen Trägheit resultiert daraus freilich auch ein Potenzial: Einmal erstelltes Kartenmaterial wird nicht - wie bei manchen Cross-Mapping-Verfahren - leichtfertig aus der Hand gegeben und erprobte Navigationsinstrumente können auch bei methodischen

1 Kurtág 2015, 22.

2 Seit dem 1967 von Richard Rorty retrospektiv beschriebenen linguistic turn z. B. der iconic, temporal, spatial, economical, ecological, religious bis zum jüngsten animal oder speculative turn, um nur eine kleine Auswahl zu nennen.

3 Die Begriffe Bedeutung und Sinn werden hier bewusst undifferenziert gebraucht und sind in etwa mit dem im Rahmen des Projekts einer allgemeinen Semiotik konturierten Sinn-Konzept gleichzusetzen (vgl. Eco 1972, 65-144).

4 Von Alexander Kluge überspitzt auf die Handlungsanweisung gebracht: „Mit der Straßenkarte von Groß-London den Harz durchwandern« (zit. nach Schulte 2005, 219). 
Weiterentwicklungen fortwirken. Und tatsächlich scheint die Musikologie der letzten Jahrzehnte in der Lage gewesen zu sein, die Impulse verschiedener Paradigmenwechsel gewinnbringend in ihr Methodenrepertoire zu integrieren (so etwa den Intertextualitätsdiskurs ${ }^{5}$ ). Nicht zuletzt konnte das auch deswegen gelingen, weil insbesondere der Musiktheorie ein notationsbasierter strukturanalytischer Ansatz als gleichsam ureigener Königsweg zu musikalischen Phänomen stets zur Verfügung stand. Dass der performative turn mit seiner Infragestellung eines textbasierten Werk- und Strukturbegriffs als besondere Herausforderung speziell auch für die Musiktheorie empfunden wird, ist daher nachvollziehbar. Dabei schließt die anti-essentialistische Bestimmung des musikalischen Werkes als «a tradition of writing and playing - and indeed listening - that is embodied in an indefinite number of variants $\ll^{6}$ eine textbetonte strukturanalytische oder traditionell hermeneutische Methodik keineswegs aus. ${ }^{7}$ Die Deklarierung des musikalischen Werks als »social construct $\aleph^{8}$ macht vielmehr darauf aufmerksam, dass eine relative, kontingente und fluktuierende Dimension musikalischer Bedeutungskonstitution existiert, welche eine flexible, immer wieder neu zu erwägende Wahl der methodischen Mittel nötig macht.

Der vorliegende Text versucht dieser Notwendigkeit zur methodologischen Selbstreflexion auf zwei Ebenen gerecht zu werden: auf einer Gegenstands- und einer MetaEbene. So soll einerseits die Musik György Kurtágs im Zentrum stehen - und zwar als spezifischer Untersuchungsgegenstand einer gleichermaßen performance-theoretisch informierten wie im Kern textstrukturell-hermeneutischen Sichtweise. Andererseits geht es aber auch um die beispielhafte Demonstration, wie methodologische Reflexion in eine konkrete analytische Betrachtung mit einfließen kann. Die übergeordnete Arbeitshypothese dabei lautet, dass dem Begriff der Interpretation, der durch die performance-theoretische Infragestellung eines normativen Werkbegriffs implizit problematisiert wird ${ }^{9}$, in doppelter Hinsicht eine wichtige Rolle zukommt: zum einen als Konzept, das den Werken und Intentionen Kurtágs konstitutiv eingeschrieben ist, zum anderen als der gemeinsame Nenner eines konkreten multiperspektivischen Analyseansatzes. Mit diesen beiden Aspekten korrespondiert auch der zweigliedrige Textaufbau. So wird im ersten Teil in kurzen, aspektweise gegliederten und stichwortartig überschriebenen Abschnitten zunächst der Befund einer Untersuchung präsentiert, welche den Korpus von Kurtágs kompositorischem Schaffen gleichsam mit performance-theoretischem Stethoskop

5 Als Beispiel einer intertextuell und analytisch fundierten musikwissenschaftlichen Studie vgl. beispielhaft Bleek 2010.

6 Cook 2013, 242.

7 Bereits die prädikative grammatische Struktur von Cooks Untertitel »Music as Performance« (Hervorhebung d. Verf.) deutet auf die inhärente Perspektivität und mithin die potenzielle Integrierbarkeit in einen multiperspektivischen Ansatz hin.

8 Cook 2013, 243.

9 Aus pragmatischen Gründen wird mit dem Interpretationsbegriff freilich auch dort noch operiert - zumeist in einem engen musikalischen, aufführungspraktischen Sinne. In seinem Buch Beyond the Score spricht Nicholas Cook an einer Stelle gleichwohl von sinterpretation, understanding that word in the broadest sense« (ebd., 79), allerdings ohne hier oder anderswo die Weite dieses Verständnisses genauer auszuloten. 
auf mögliche Resonanzräume eines erweiterten Interpretationsbegriffs hin abtastet. Der zweite Teil dann widmet sich der konkreten analytischen Betrachtung eines Komplexes von zwei Werken Kurtágs: Samuel Beckett: What is the Word op. 30a für Stimme und Pianino (1990) sowie Samuel Beckett: What is the Word op. 30b für Rezitation, Stimmen und im Raum verteiltes Kammerensemble (1991). In drei getrennten Durchläufen wird dabei jeweils ein Paradigma - (Inter-)Textualität, Medialität, Performativität - als methodische Grundorientierung gewählt.

\section{ASPEKTE VON INTERPRETATION IM SCHAFFEN KURTÁGS}

\section{Stellenwert}

»Sprache interpretieren heißt: Sprache verstehen; Musik interpretieren: Musik machen. ${ }^{10}$ Das in diesem Diktum Theodor W. Adornos beispielhaft formulierte Verständnis von musikalischer Interpretation als primär ausführendem Vollzug eines Notentextes ${ }^{11}$ nivelliert die konnotative Vielschichtigkeit des musikalischen Interpretationsbegriffs, die bereits durch einen kursorischen Blick in die Begriffsgeschichte aufgezeigt wird. So ist seit seinem Aufkommen im 19. Jahrhundert im Begriff der musikalischen Interpretation stets eine Spannung zwischen hermeneutisch-deutenden und darstellend-praktischen Momenten präsent ${ }^{12}$, welche im Ergebnis dafür sorgt, dass sich die Kategorie der Interpretation «so wenig im Begriff des gemeinhin als Interpret bezeichneten ausführenden oder reproduzierenden Künstlers [erschöpft] wie die Kategorie der Rezeption im Begriff des Hörers. «" ${ }^{13}$

Daher gilt es, die Kategorie ıInterpretation` auch im Kontext der Musik György Kurtágs potenziell in der gesamten Bandbreite ihres semantischen Bedeutungsspektrums zu fokussieren - umso mehr, als ihr der Komponist selbst einen außerordentlichen Stellenwert für sein kompositorisches Schaffen zuspricht. Dass die im Motto dieses Textes zitierte Aussage, sein Komponieren sei weniger auf das klingende Resultat ausgerichtet, denn als Medium seiner Interpretationsauffassung anzusehen - bei aller zuweilen an Koketterie grenzenden Bescheidenheit des Komponisten - beim Wort zu nehmen ist, macht bereits der in der Rezeption seines kompositorischen Wirkens häufig vernachlässigte Umstand deutlich, dass Kurtág in seiner gesamten musikalischen Karriere niemals Komposition unterrichtete, sondern - von 1967 bis 1993 als Dozent an der Budapester Franz-Liszt-Musikakademie - alleine Kammermusik-, Klavier- und Interpretationsklassen

10 Adorno 1956/78, 253.

11 Was im Kontext von Adornos pointierter Gegenüberstellung als bewusste Zuspitzung eines andernorts deutlich differenzierter explizierten Interpretationsverständnisses gelten muss, kann als Grundspannung zwischen einem einerseits sprachlich geleiteten und andererseits musikalischpraktischen Zugang zu Musik freilich bis in jüngere Ansätze nachvollzogen werden (so etwa bei Wellmer 2009).

12 Vgl. Danuser 1992, 452.

13 Ebd., $449 f$. 
gab - und das offenbar mit so nachhaltig prägender Wirkung, dass daraus langjährige künstlerische Partnerschaften mit ehemaligen Student*innen wie etwa Zoltán Kocsis oder dem Keller-Quartett entstanden. Seine Abstinenz vom aktiven Kompositionsunterricht begründet Kurtág damit, dass »es vielleicht doch besser ist, die Muster und Methoden nicht zu gut zu kennen, um sich die nötige Frische bewahren zu können und nicht zu geschickt zu werden «. ${ }^{14}$ Das Unterrichten von praktischer Interpretation hingegen sei für ihn, so bemerkt Kurtág in einem Fernsehinterview, »a very egoistic thing ${ }^{15}$, denn »| understand really music only, if I teach it. $\aleph^{16}$ Der Schluss, dass nicht nur Kurtágs allgemeines Musikverständnis, sondern auch seine Komponierpraxis eng mit seiner Auffassung von Interpretation zusammenhängt, liegt daher durchaus nahe. Wie genau die erwähnten »Ideen von Interpretation « aussehen, lässt Kurtág freilich nur indirekt erkennen, so etwa wenn er in einem anderen Interview folgende grundlegende Forderung gegenüber Musiker*innen ausspricht: »They must make an effort to understand what they are about to play and understand its structure. $^{17}$ Dieses strukturanalytisch-hermeneutische Gebot wird durch den wenig später aufgerufenen Topos des Sprachcharakters von Musik (»Music is a language, it is like talking $\aleph^{18}$ ) gleichzeitig zur Grundlage einer allgemeineren kommunikationstheoretisch-semantischen Konzeption von Interpretation. Dass darüber hinaus keine genauere Eingrenzung von Kurtágs allgemeinem Interpretationsverständnis möglich scheint, verweist dabei gerade auf dessen Kern, wird Interpretation doch primär als eine Praxis begriffen, deren Potenzial erst im aktualisierenden Vollzug annähernd ausgemessen werden kann - und zwar gleichermaßen im vermittelnd-lehrenden und musikalisch-ausführenden wie - so die im Folgenden aspektweise auszudifferenzierende These - im kompositorisch-praktischen Vollzug.

\section{Spiel}

Der Begriff des Spiels liegt für die performance-theoretische Annäherung an Kurtágs Musik nahe, mehr noch: Er drängt sich geradezu auf, denn sowohl in Kurtágs Schaffen als auch in den performance studies nimmt das Spielkonzept eine prominente Stelle ein. So ist es, nachdem es zuvor schon in anderen Theoriekontexten des 20. Jahrhunderts eine beachtliche Karriere durchlaufen hat ${ }^{19}$, im performance-theoretischen Diskurs sowohl als Denkfigur (»Playing [...] is at the heart of performance $\aleph^{20}$ ) wie auch als konkreter Untersuchungsbereich bzw. zentrale Fragestellung zu einem wichtigen Bezugspunkt geworden (»What do playing baseball, [...] playing a role in a play, playing the fool, [...]

14 Kurtág 1997, 28.

15 Kele 1996, 17:42-17:48.

16 Ebd., 17:13-17:16.

17 BBVA Foundation 2015, 0:20-0:31.

18 Ebd., 0:40-00:42.

19 Beispielsweise in der Kulturanthropologie Johan Huizingas, der Sprachphilosophie Ludwig Wittgensteins oder der Hermeneutik Hans Georg Gadamers.

20 Schechner 2002, 89. 
and playing out an idea have in common? $\aleph^{21}$ ). Und in Kurtágs Musik sticht das Wort sSpiek bereits in zahlreichen Stücktiteln hervor, etwa in der losen und für verschiedene Besetzungen geschriebenen Sammlung Signs, Games and Messages (1989-98) oder am bekanntesten - in Játékok (seit 1973), der Sammlung von »Spielen für Klavier«, die - an Béla Bartóks Mikrokozmosz (1926-39) anschließend - sowohl ein pädagogisches Klavierlehrwerk als auch ein kompositorisches Laboratorium und work in progress darstellen. Das Vorwort zum ersten Band ist für Kurtágs Verständnis von Spiel und Spielen mehrfach aufschlussreich, denn dort heißt es:

Spiel ist Spiel. Es verlangt viel Freiheit und Initiative vom Spieler. Das Geschriebene darf nicht ernst genommen werden - das Geschriebene muß todernst genommen werden: was den musikalischen Vorgang, die Qualität der Tongebung und der Stille anlangt. ${ }^{22}$

Neben der für Kurtág ungewohnten rhetorischen Qualität (die Tautologie am Anfang, die Sustentio der klimaktischen Pointe sernst - todernst`) fallen daran zwei Aspekte ins Auge: Zum einen scheint aus der Betonung des Bezugs auf den Notentext die bereits oben angedeutete Strenge hinsichtlich strukturellen Denkens und sprachähnlicher Logik zu sprechen. Zum anderen entsteht aber auch eine seltsame Spannung, denn unmittelbar vorausgehend wird auf die für die Ausführung der Stücke nötige Spielinitiative verwiesen, ohne allerdings aufzulösen, wie ein Ausgleich zwischen den beiden gegenläufigen Kräften `Strenge` und `Gestaltungsfreiheitı aussehen könnte. Zusätzlich verschärft wird diese Spannung durch die Ausbuchstabierung der Bezugsgrößen für die geforderte Ernstnahme des Notentextes; genannt werden nämlich ausgerechnet solche Parameter und Gestaltungsdimensionen, die nur eingeschränkt, vermittelt bzw. nicht eindeutig notiert sind (der »musikalische Vorgang», der sich z. B. aus der formalen Dramaturgie erschließt; die "Qualität der Tongebung", die durch Artikulationsangaben nur partiell determiniert und wesentlich etwa durch den Stückcharakter suggeriert wird; die "Stille», die nicht gleichzusetzen ist mit notierten Pausen, welche wiederum in Kurtágs eigenwilliger und häufig verwendeter relativer Notation nicht exakt festgelegt sind ${ }^{23}$ ). Mit anderen Worten: Das „Geschriebene [...] todernst « zu nehmen, ist nicht gleichbedeutend mit der Decodierung der diskreten Zeichenfolge des Notentexts; dessen ernsthafte Lektüre geht vielmehr damit einher, dass immer schon komplexe synthetische Entscheidungen darüber getroffen werden, was der Notentext für die musikalische Ausführung bedeutet. Dass dennoch von sSpielen nicht zu vernachlässigende Differenz an, dass der Akt des Interpretierens zumindest partiell eine bewusste und reflektierte Entscheidungsfindung erfordert, während die Eigendynamik des Spiels oder - um mit Hans Georg Gadamer zu sprechen - »das Spiel als Vollzug der Bewegung als solcher ${ }^{24}$ die Schwere subjektiver Entscheidungsfindung

21 Ebd., 91.

22 Kurtág 1979, Vorwort, o.S.

23 Ebd.

24 Gadamer 1960, 109. 
aufhebt und vergessen macht. Im ersten Band der Játékok entspricht dieser Differenz tendenziell das Binnenverhältnis der auf einer Doppelseite in Opposition gebrachten sSpiek-Stücke: Während das linke Stück in relativ voraussetzungslos zugänglicher, ‘grob aufgelöster graphischer Notation gehalten ist und damit ein intuitives Spiel begünstigt, erfordert das rechte Stück in konventionellerer Notation die Einhaltung und im Zweifelsfall auch Reflexion traditioneller Verhaltenscodes musikalischer Ausführungshandlungen. Als verbindendes, den Spielcharakter der Stücke garantierendes Element fungiert gleichwohl in beiden Fällen die graphisch-sinnliche, wortwörtlich augenfällige Qualität der Notation. Ihre relative Unmittelbarkeit suggeriert eine Einfachheit, die sich gleichsam als vorinterpretative Verspieltheit in der Ausführung aktualisieren kann. Musikalisches Spielen im Kurtág'schen Sinne könnte man also als eine Voraussetzung oder Propädeutik von Interpretationspraxis begreifen, ohne dass jenes von dieser vollkommen geschieden wäre, denn in jedem interpretatorischen Vollzug ist ein spielerisches Moment, mehr oder weniger untergründig, enthalten.

Die aufgezeigte Konstellation von interpretatorischen und spielerischen Momenten wird noch deutlicher, wenn man die Funktion der Játékok für Kurtágs Musik allgemein in den Blick nimmt. So hat Mieke van Hove detailliert nachgewiesen, dass Kurtágs ıSpiele«Sammlung in besonders enger und ganz unterschiedlicher Weise mit dem übrigen Schaffen Kurtágs verwoben ist: ${ }^{25}$ als personalisiertes Studienmaterial für seine Schüler ${ }^{26}$, als Keimzelle, Experimentierfeld und Materialsammlung für andere Kompositionen und - als über Jahrzehnte entstehendes work in progress - auch in allgemeinerer Form als Medium der kompositorischen Selbstverständigung. Das intime Verhältnis von Spiel und Interpretation lässt sich also nicht alleine auf die Perspektive der ausführenden Musiker der Játékok bzw. von Kurtágs Stücken allgemein, sondern auch auf Kurtágs Komponieren selbst beziehen. ${ }^{27}$

Ein biographischer Umstand darf in diesem Zusammenhang nicht unerwähnt bleiben: In Kurtágs Laufbahn hat es bekanntlich immer wieder Phasen gegeben, in denen er nur unter großer Mühe oder teilweise auch gar nicht zu komponieren in der Lage war, was sich bis heute in einem dezidiert skrupulösen und selbstkritischen Verhältnis zum eigenen Komponieren widerspiegelt. Spielerische Zugänge zum Komponieren, die Kurtág während seiner größten Schaffenskrise in den Jahren 1957/58 in Paris von der Psychologin Marianne Stein dadurch nahegebracht wurden, dass diese ihm radikal reduzierte Kompositionsaufgaben stellte (eine Komposition mit zwei Tönen und Ähnliches) ${ }^{28}$, können daher auch als Bewältigungsstrategien für die individuelle Schaffensproblematik

25 Vgl. Hove 2007.

26 Als jüngst zum ersten Mal publiziertes Beispiel ist hier die handgeschriebene Version einer Auswahl von Játékok-Stücken anzuführen, die Kurtág für seinen Klavierschüler Zoltán Kocsis angefertigt hat und die in zahlreichen Details von der soffiziellen` Notenausgabe der Játékok abweicht (vgl. Kurtág 2016).

27 Vgl. dazu auch Hohmaier 2001. Dort wird u.a. anschaulich gezeigt, wie sich Kurtág fremdes Material, in diesem Fall eine Passage aus Bartóks Zweitem Streichquartett, gleichermaßen spielerisch wie analytisch aneignet und in die Sphäre des eigenen Komponierens überführt.

28 Vgl. Kurtág/Dibelius 1997, 31. 
angesehen werden. ${ }^{29}$ Dem Klavier kommt dabei eine besondere Rolle zu. Denn als ausgebildeter Pianist hat Kurtág zu dem Instrument ein naturgemäß besonders enges, sinnlich-haptisches Verhältnis aufgebaut. Das schlägt sich in verschiedener Weise nieder: In zahlreichen Kompositionen ist es nicht nur prominent eingesetzt, auch wird das verwendete musikalische Material wesentlich durch die Spezifik des Instruments geprägt, z. B. im Fall der den gesamten Tonraum des Klaviers durchmessenden absteigenden CDur-Tonleiter im ersten Satz von ...quasi una fantasia... op. 27/1 für Klavier und im Raum verteilte Instrumentalgruppen (1987/88). ${ }^{30}$ Daneben dient Kurtág das Klavier aber auch als Medium des Komponierens selbst. So berichtet er, dass er einmal Komponiertes stets am Klavier überprüft. ${ }^{31}$ Bemerkenswerterweise kommt dieser ersten klanglichen Aktualisierung des Notierten dabei oft eine Art Originalfunktion zu, denn häufig passiere es, dass Stücke

in der Weise wahr sind, wie ich sie auf dem Klavier spielte, als ich sie komponierte, und wie ich sie nie wieder so darstellen kann. Es ist wie ein Traum - eine wunderbare Musik, und wenn ich sie aufschreibe, stellt sich heraus, dass es nicht so wunderbar ist. ${ }^{32}$

Gegenüber der Reproduzierbarkeit des Notentextes kann die Einzigartigkeit und NichtWiederholbarkeit des Spiels also zuweilen sogar den Primat beanspruchen. Das spielerische Moment wird damit nicht nur, wie oben gesehen, zu einem integralen Bestandteil des rezeptiv-interpretatorischen Zugangs zu Musik, sondern, als gleichsam originär schöpferische Interpretation, auch zum Idealbild oder - in der von Kurtág im obigen Zitat aufgerufenen Metaphorik - sTraumbild von Komponieren schlechthin.

\section{Form}

Kurtágs Formdenken zeichnet sich durch eine abgezirkelt-komprimierte Formulierungskunst aus, welche in neuerer Musik - wie wiederholt bemerkt worden ist - wohl nur mit der apodiktischen Kürze Anton Weberns verglichen werden kann. Sowohl in Kurtágs Zyklen (etwa in den Botschaften des verstorbenen Fräulein R. V. Trussova für Sopran und Kammerensemble op. 17, 1976-80, oder den Szenen aus einem Roman für Sopran, Violine, Kontrabass und Cimbalom op. 19, 1979-82) als auch in den offeneren StückSammlungen (wie Signs, Games and Messages, Játékok, ... pas à pas - nulle part... für Bariton solo mit und ohne Instrumente op. 36, 1993-97) kommt sie ganz unmittel-

29 In diesem Kontext sei auch noch einmal an die oben erwähnte Skepsis Kurtágs gegenüber der Befolgung von handwerklichen Regeln und Mustern erinnert, in der sich ebenfalls - ex negativo - eine Affinität zu einem einfach-spielerischen, nicht allzu reflexionsschweren Komponierzugang ausdrückt.

30 Für diese Stücke gilt in besonderer Weise, was Gösta Neuwirth treffend am Anfangsmotiv von Kurtágs Solobratschen-Komposition Panaszos nóta (1987/91) aus Signs, Games and Messages beobachtet, nämlich dass »das Technische, die Ausführung, der Interpret, von vornherein mit hineinkomponiert, als Form und Inhalt verschränkt sind« (2011, 23).

31 Vgl. Kurtág 2010, 50.

32 Ebd. 
bar zur Geltung, nämlich in der Abfolge von kurzen Einzelstücken, quasi monadischen Einheiten, welche selten länger als zwei oder drei Minuten dauern. Aber auch in groß dimensionierten einsätzigen Werken (etwa dem Doppelkonzert für Klavier, Violoncello und zwei Ensembles op. 27/2, 1989/90, oder in ... concertante... für Violine, Viola und großes Orchester op. 42, 2003) lässt sich die Gesamtform in der Regel unschwer als Sequenz kurzer Formabschnitte analysieren. Musikalische Dramaturgien sind daher auch nicht als Resultat formal-architektonischer, kohärenzbasierter Planung anzusehen, sondern emergieren als übergeordnete Zusammenhänge aus der Konstellation der Einzelteile. Die einer solchen Formbildungslogik inhärente Zentripetalkraft macht sich speziell in den umfangreicheren Werken des oben erwähnten zweiten Typus (Stück-Sammlungen) als Dynamik zu einer Art offenen Großform bemerkbar. In einigen Werken hat das zur Konsequenz, dass Kurtág, da er »keine Möglichkeit und keinen Sinn für eine alleinseligmachende lineare Ordnung sieht ${ }^{33}$, alternative Lösungen der großformalen Disposition wählt. So basiert die letztendliche Abfolge der Kafka-Fragmente für Sopran und Violine op. 24 (1985-87) auf einem Vorschlag des Musikwissenschaftlers und ehemaligen Kurtág-Studenten András Wilheim. ${ }^{34}$ In einem anderen Fall, der Sammlung Einige Sätze aus den Sudelbüchern Georg Christoph Lichtenbergs für Gesang mit verschiedenen Instrumenten op. 37 (1996/99), geht Kurtág noch einen Schritt weiter, indem er ein Repertoire von aphoristischen Einzelnummern anbietet, aus dem die ausführenden Musiker*innen die Auswahl und Reihenfolge der zu spielenden Stücke bestimmen sollen. Durch die Delegation der großformalen Anlage an die musikalischen Interpret*innen werden diese konzeptionell zur mitschöpfenden Instanz erhoben und die Grenzen zwischen Komposition und Interpretation aufgeweicht bzw. tendenziell in ein Kontinuum überführt. Dass hinter dieser formalen Öffnungsstrategie allerdings eine dezidierte kompositorische Haltung steht, machen Kurtágs vorangestellte Erläuterungen in der Druckausgabe von op. 37 klar. Dort heißt es:

Die Interpreten sollten für die Aufführung im Konzert eine Auswahl treffen - mithin ihren eigenen Zyklus komponieren. [...] Bei der Auswahl und Anordnung sollte man sich von dem Gesichtspunkt leiten lassen, dass die Charaktere und die Tonarten miteinander kontrastieren und sich ergänzen. ${ }^{35}$

Die explizite Formulierung »ihren eigenen Zyklus komponieren « und die Vorgabe von Ordnungsprinzipien (Komplementär- und Kontrastbildung) machen deutlich, dass die entstehende Gesamtform als logischer Sinnzusammenhang intendiert ist, der von seinen Interpret*innen explizit kompositorisch-reflektiertes Formdenken abverlangt. Eine solche Verantwortungsübertragung kann aus Komponistensicht freilich auch das Risiko bergen, dass die entstehende Großform nicht überzeugt. Und in der Tat scheint für den Fall von op. 37 - zumindest für Kurtág - nicht zuzutreffen, was Wilheim am Beispiel der KafkaFragmente behauptet, nämlich dass in Bezug auf die Zusammenstellung der Einzelstücke

33 Wilheim 1997, 38.

34 Vgl. Kurtág 1997, 28.

35 Kurtág 1996, o.S. 
»unzählige Weisen der Zusammengehörigkeit vorstellbar« seien, wobei »selbst bei den scheinbar am wenigsten plausiblen Auffassungen lediglich die Glaubwürdigkeit der Wiedergabe eine Grenze [setzt] «36. Denn dass Kurtág die ursprüngliche Druckausgabe von op. 37 zurückgezogen hat und stattdessen nur noch eine als op. 37a vom Verlag vertriebene Version angeboten wird, deren Stückzusammenstellung von Kurtág selbst festgelegt ist ${ }^{37}$, zeigt an, dass aus Sicht des Komponisten der performativen Überzeugungskraft der Interpretation offensichtlich doch eine Grenze gesetzt ist.

Einen Grund für die fehlende Realisierung von Kurtágs Konzept der interpretativ zu komponierenden Großform sieht Michael Kunkel in »Kurtágs starke[r] kompositorische[r] Fixierung auf einzelne Interpreten ${ }^{38}{ }^{38}$ Und tatsächlich scheinen - zumindest in manchen der langjährigen künstlerischen Musiker*innen-Partnerschaften Kurtágs ${ }^{39}$ - sich arbeitsteilige Rollenmuster zu manifestieren. Wenn man das Konzept der Partitur als social script $t^{40}$ - von Cooks Intention losgelöst - wörtlich nimmt, würde in diesen Fällen Kurtág also in der Rolle des Regisseurs verbleiben. Besonders deutlich zeigt sich das auch an der von Kurtág ebenfalls im Vorwort von op. 37 skizzierten Möglichkeit der Interpreten, nicht nur die Reihenfolge der angebotenen Stücke zu bestimmen, sondern diese auch in einem »komponierten Konzertprogramm « ${ }^{41}$ mit Musik anderer Komponisten zu kombinieren. Bezeichnenderweise ist das Konzept eines solchen komponierten Programms bisher - in einem anderen Kontext ${ }^{42}$ - nur von Kurtág selbst erfolgt. Im Hiatus zwischen konzeptioneller Offenheit und determinierender Praxis tritt also eine Spannung an die Oberfläche. Genauer charakterisierbar ist sie durch die Dichotomie zwischen rationalbegründbaren und intuitiv-gefühlten Entscheidungsfindungen. So verlangt Kurtág auf der einen Seite von seinen Interpret*innen bewusste kompositorisch-logische Reflexion, auf der anderen Seite handelt er bei seinen eigenen kompositorischen Festlegungen eher wie ein Interpret, der, ohne explizite Gründe angeben zu können, intuitiv die für richtig erachteten Entscheidungen trifft. Diese Divergenz tritt in der Zusammenarbeit von Kurtág mit seinen musizierenden Interpret*innen freilich selten offen zutage, weil im Zweifelsfall der Komponist als lenkende Interpretationsinstanz akzeptiert wird. Zu fragen bleibt, wie sich das skizzierte Spannungsverhältnis auf die musikalische Realisierung auswirkt, wenn der Komponist weder als interpretierender noch als kommentierender Exeget seiner eigenen Musik in Erscheinung tritt. Das zu erweisen bedürfte es, wie Michael Kunkel zu

36 Wilheim 1997, 38.

37 Ähnliches gilt für ...pas à pas - nulle part... für Bariton, Streichtrio und Schlagzeug op. 36 (1993-97): Auch hier war eigentlich eine offene Form konzipiert, bisher wurde das Werk allerdings nur in der vom Komponisten festgelegten ,Pariser Fassung، der Uraufführung gespielt.

38 Kunkel 2008, 155.

39 So weist etwa die Sängerin Adrienne Csengery die Insinuation, sie sei eine 'Mitschöpferin von Kurtágs Werken, weit von sich (vgl. Csengery/Balázs 1986, 57).

40 Vgl. Cook 2013, 249-256.

41 Kurtág 1996, Vorwort, o.S.

42 Zusammen mit seiner Frau Márta hat Kurtág seit einem 1993 in Salzburg stattgefunden Porträtkonzert wiederholt ein 'komponiertes` Klaviermusik-Programm zur Aufführung gebracht, das aus immer wieder neu variierten Konstellationen von Játékok-Stücken und eigenen vierhändigen Arrangements verschiedener Werke J.S. Bachs besteht. 
Recht bemerkt, »einer regen Aufführungspraxis mit Interpreten, die genug Mut aufbringen müssten, den ordnenden Willen des Autors nach Redaktionsschluss zu missachten.

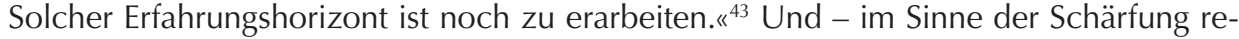
flektierter interpretativer Entscheidungskompetenz - wäre zu ergänzen: Auch der Erfahrungshorizont einer musiktheoretischen Sichtweise, welche mit analytischen Methoden gleichermaßen als Anwalt wie als Kritiker des Autorwillens auftreten kann, ist noch zu erarbeiten.

\section{Prozess}

Ein Argument für die am Ende des letzten Abschnitts angeregte verantwortungsvolle Befreiung von determinierenden Interpretationsvorgaben Kurtágs kann in einem allgemeinen Merkmal von Kurtágs Schaffen gefunden werden, welches sich am ehesten auf den Begriff des Prozesses bringen lässt. So ist in Kurtágs Musik erstens ein prominenter produktionsästhetischer Prozesscharakter zu konstatieren. Denn auch wenn in formalstruktureller Hinsicht Kurtágs Stücke mehrheitlich eher einem Moment- als einem Prozesstypus zuzuordnen sind, kann die Form ihrer Genese in beide Kategorien fallen. Auf der einen Seite stehen demnach Stücke, die - oft in sehr kurzer Zeit und in einem Wurf - skizziert bzw. niedergeschrieben und ohne große Veränderungen von Kurtág für gültig befunden werden; der anderen Seite zuzuordnen sind jene Kompositionen - und sie stellen die Mehrheit -, welche einen längeren Entstehungsprozess mit zahlreichen Korrektur- und Überarbeitungsschritten durchlaufen. ${ }^{44}$ In letzterem Fall wird Komponieren zu einem performativen Vollzug, der in seiner durch Selbstbefragung und Verbesserungsbemühung geleiteten Repetitionsstruktur dem Übeprozess eines Musikers ähnelt. ${ }^{45} \mathrm{Er}$ wähnens- und bemerkenswert ist dieses - in ähnlicher Weise natürlich auch bei anderen Komponist*innen zu findende - prozessuale ,Überschreibungskomponieren im Fall von Kurtág deswegen, weil es mit dem zweiten prozessdominierten Aspekt in Kurtágs Musik konfligiert. Dieser betrifft die Ebene der Erarbeitung der Kompositionen, welche - wie bereits erwähnt - sehr oft von Kurtág selbst begleitet oder angeleitet wird, insbesondere wenn er mit langjährig bekannten Interpret*innen zusammenarbeitet. ${ }^{46}$ Wie von unterschiedlicher Seite berichtet worden ist ${ }^{47}$, kommt es im Laufe eines solchen Erarbeitungsprozesses häufig dazu, dass Kurtág Modifikationen am Notenmaterial seiner Komposition vornimmt. Diese können von eher marginalen Ergänzungen des Notentexts (z. B. Eintragungen von Stricharten) bis zu signifikanten Veränderungen der Partitur reichen

43 Kunkel 2008, 155.

44 Vgl. Kurtág 2015, 26.

45 Freilich mit dem Unterschied, dass es beim Komponieren in der Regel keinen präterminierten, der musikalischen Aufführung vergleichbaren Enddurchlauf gibt.

46 Der für zeitgenössische Musik untypische Umstand, dass Kurtág die Aufführungsrechte von einigen seiner Kompositionen für spezifische Interpreten reserviert hat (so bei ... concertante... op. 42 und auch beim später zu besprechenden op. 30a/b), zeigt an, dass ihm ein gründlicher Erarbeitungsund Einstudierungsprozess seiner Stücke sehr viel wichtiger ist als ihre Aufführung um jeden Preis (erinnert sei hier auch noch einmal an das Kurtág-Zitat des Mottos).

47 Vgl. Kurtág 2010, 42f. sowie Csengery/Balázs 1986, 56-58. 
(wie etwa die Hinzufügung oder Transposition einzelner Töne oder ganzer Passagen und Melodielinien). Der Notentext - selbst Ergebnis eines oft mühevollen Kompositionsprozesses - tritt also in seiner Bedeutung zuweilen hinter die im Erarbeitungsprozess emergierende Stimmigkeit einer Interpretation zurück. Die Sängerin Adrienne Csengery fasst die hinter dieser Flexibilität Kurtágs stehende Motivation als gleichsam personalisierte Prozessorientierung zusammen: „Von Belang ist für ihn [Kurtág] einzig und allein, ob ich mich auf dem richtigen Weg befinde zum Ziel hin, das er uns gesteckt hat.« ${ }^{48}$

Aus der geschilderten Interpretationspraxis Kurtágs lassen sich für die Zwecke dieser Untersuchung zwei Schlüsse ableiten: Zum einen wird die hier vertretene These des engen Bezugs von Komposition und Interpretation samt der tendenziellen Priorisierung der Interpretationskomponente unterstrichen. Die enge Bindung Kurtágs an individuelle Interpreten lässt zuweilen sogar den Eindruck von kompositorisch-interpretativen Autorschaftssymbiosen entstehen. Zum anderen wird das wechselseitige Verhältnis von Komposition und Interpretation aber auch problematisiert. Das wird vor allem immer dann deutlich, wenn dem musikalischen Interpreten nicht mehr alleine - wie im Fall des von Wilheim treffend als »Kaleidoskop in klassischem Rahmen « ${ }^{49}$ charakterisierten op. 37 - eine Freiheit innerhalb eines klar umrissenen Spielfelds gewährt wird, sondern wenn die Grenzen dieses Spielfelds, die Textualität der Partitur, durch die Interpretationspraxis selbst signifikant modifiziert werden. Denn auf diese Weise wird eine Rahmensetzung, die für Kurtág wie für die meiste zeitgenössische Musik nach wie vor konstitutiv ist, prinzipiell infrage gestellt. Dass das einhergehende Problem der Werkidentität im Fall von Kurtágs Musik nicht akut wird, hängt allein mit der unhinterfragten Autorität des Komponisten zusammen, sein Werk notationell zu verändern. Gleichzeitig wird deutlich, dass die oben unterstellte kollaborative Gleichberechtigung von Komponist und Interpret*in in der Praxis nicht konsistent verwirklicht ist, sondern dass den Interpret*innen vielmehr nach wie vor ein subordinierter smedialer Status zukommt. ${ }^{50}$

Die hier aufgeworfene Problematik betrifft - mutatis mutandis - freilich auch den analytischen Zugang zu Kurtágs Musik: Wenn - wie von einer performance-theoretischen Position ja generell und in vielen Fällen unmittelbar plausibel angemerkt - der Notentext nicht mehr der alleinige Referenzpunkt einer Analyse sein kann, was sollte an seine Stelle treten: spezifische Aufführungen oder Aufnahmen? Selbst bei deren Untersuchung wäre im Normalfall das Verhältnis von interpretativer klanglicher Aktualisierung und notentextueller Vorlage nur von einer Seite her flexibel, ist der Notentext - sieht man einmal von editionstechnischen und anderen textuellen Varianten $a b$ - in der Regel doch konstant; wenn aber auch die Textgrundlage in ihrer Gültigkeit zumindest potenziell infrage steht, begibt man sich unweigerlich aufs analytische Glatteis. Eine andere Alternative wäre, von Kurtág initiierte Veränderungen des Notentextes als legitime Variantenbildungen einzustufen, sie also als gültige und mithin einer Analyse gleichermaßen zugängliche Versionen des Stücktextes anzusehen. Das wäre methodisch

48 Ebd., 57.

49 Wilheim 1997, 38.

50 Vgl. dazu die Sängerin Adrienne Csengery: »Ich also schenkte ihm meine Stimme, wie einem Bildhauer ein Stück Holz, an dem er dann schnitzen konnte« (Csengery/Balázs 1986, 55). 
gesehen zwar kohärent, würde aber einerseits den dezidiert personalisierten, auf spezifische Interpreten zugeschnittenen Charakter der Veränderungen verkennen und - durch die Akzeptanz der exzeptionellen Komponistenstellung - andererseits einem einseitig biographistischen Deutungsmuster Vorschub leisten. ${ }^{51}$ Oder - ein letzte, fraglos leicht abseitige, von dem am Ende des vorletzten Abschnitts angeführten Kurtág-Zitat aber durchaus gedeckte Variante - sollte gar die von Kurtág implizierte klanglich-aktualisierte `Originalgestalt ‘ gleichsam als platonische Idee des Werks zum imaginären Zielpunkt der analytischen Bemühungen werden? Das würde freilich akute theoretisch-idealistische Komplikationen mit sich bringen und das aus dem Strukturalismus bekannte Problem der Suche nach einer 'Struktur hinter der Struktur a aufwerfen. Kurz: Eine Antwort auf das aufgezeigte methodische Dilemma scheint zu diesem Zeitpunkt nicht möglich. Den Wink für einen möglichen Ausweg könnte ein weiteres mit der Interpretationsthematik in engem Verhältnis stehendes Konzept in Kurtágs Musik liefern, welches im Folgenden beleuchtet werden soll.

\section{Botschaft}

Die Anzahl von Widmungen und Hommagen, Zitaten und Anspielungen in Kurtágs Musik ist nahezu unüberschaubar; es gibt kaum ein Stück, das nicht in irgendeiner (sei es klanglich nachvollziehbaren oder paratextuellen) Form Bezüge signalisiert: zu anderer Musik, eigener Musik, geschätzten Künstlerkolleg*innen (lebenden wie verstorbenen) oder - vor allem - Freunden und Weggefährten. Diese referenzielle Omnipräsenz kann auf einer basalen produktionstechnischen Ebene als Resultat einer Strategie gedeutet werden, welche produktive Hemmnisse durch die Konkretion persönlicher Adressierungen oder Veranlassungen zu überwinden hilft. Darüber hinaus ist sie für Kurtág aber auch Sinnstiftung für sein Komponieren schlechthin. Das kommt in einer Aussage des Komponisten zum Ausdruck, in der er - unter Nennung von Interpretin und Adressatin einer Widmungskomposition - das für ihn zentrale und auch in zahlreichen seiner Stücktiteln zu findende Konzept der Botschaft beispielhaft umschreibt: »Das Komponieren ist strikte Privatsache. Was ihm Sinn gibt, ist, dass Jutka Horváth aufbrach und Józsa Blum das Stück vorspielte. Das ist eine Botschaft « ${ }^{52}$. Eine Botschaft zeichnet sich für Kurtág also durch eine mediale Komponente aus (sie ist von einem Sender, hier György Kurtág, an einen Empfänger, Józsa Blum, adressiert und wird von einer dritten Instanz, Jutka Horváth, übermittelt ${ }^{53}$ ) und gleichzeitig weist sie ein performatives Moment auf (sie wird mithilfe eines Mediums aktualisiert, in diesem Fall von der Musikerin, die Kurtágs Kompositionstext interpretiert). In Kurtágs Beispiel ereignet sich all das freilich in einem streng personalisierten und privaten Rahmen. Sinn und Inhalt der Botschaft sind, wenn

51 Als gedankenexperimentelle Gegenprobe ließe sich z. B. fragen: Was, wenn die exakt selben Textveränderungen nicht von Kurtág, sondern von dem/der Interpret*in selbst getroffen worden wären - müssten sie dann in gleicher Weise als gültige Versionen angesehen werden?

52 Kurtág 2010, 50.

53 Das Übermittlungsmoment wird im Zitat durch die explizite Erwähnung des »Aufbruchs«, also einer medialen Übertragungsbewegung hervorgehoben. 
überhaupt, nur dem Autor und der Adressatin (evtl. auch der Überbringerin) zugänglich, nach außen hin würde sie hermetisch bleiben (auch schon deshalb, weil in diesem wie in zahlreichen anderen Fällen von Widmungs- und Botschaftskompositionen Kurtágs die Adressat*innen dem engsten Umkreis des Komponisten entstammen, für die Rezipienten also gewissermaßen Leerstellen sind). Gleichwohl ist es ja gerade die Komposition selbst, die explizit als >Botschaft gekennzeichnet ist und deshalb auch in öffentlichen Kommunikationssituationen - wenn sie beispielsweise (sei es von Jutka Horváth, sei es von anderen Musiker*innen) in einem Konzert gespielt wird - ihren Botschaftscharakter bewahren müsste. Die in Kurtágs Zitat implizierte formale Beschreibung der Botschaft sagt also nichts über deren Inhalt aus: Sie gibt (aus Autorperspektive) zwar dem Komponieren Sinn, sie ist (aus Rezipient*innenperspektive) aber nicht der Sinn der Komposition selbst.

Doch worin könnte nun der Sinn der Botschaft bzw. - wenn man den Botschaftsbegriff metonymisch begreift - die kommunikative Qualität von Kurtágs Musik allgemein begründet sein? Ein naheliegender und daher auch immer wieder praktizierter analytischer Beantwortungsansatz dieser Frage besteht darin, die Sinnsuggestionen der Komposition gleichsam beim Wort zu nehmen und zur Sprache zu bringen, indem erkennbare Referenzen gesucht, aufgeschlüsselt und zueinander in Beziehung gesetzt werden. Ein solches 'klassisch hermeneutisches` Vorgehen funktioniert naturgemäß immer dann besonders gut, wenn im untersuchten Gegenstand textuell-sprachliche Elemente involviert sind, beispielsweise im Fall von Vertonungen, bei denen die musikalische Bedeutungsebene auf die Semantik der Textvorlage bezogen werden kann. Und tatsächlich kommt man mit dieser Methodik in Kurtágs Vokalmusik oft schon recht weit, denn diese - so wird von Kunkel zu Recht bemerkt - »befindet sich grundsätzlich in einem abbildenden Verhältnis zur Sprache der herangezogenen Texte $\aleph^{54}$. Aus der Analyse der Wort-TonBeziehung lassen sich also, selbst wenn das »mimetische [...] Spektrum [...] von der klanglichen Illustration bis zum Vollzug einer Textidee im Tonsatz ${ }^{55}$ sehr weit reichen kann, zumindest die Grundzüge eines text-musikalischen Sinngehalts erschließen. Komplizierter wird es, wenn sprachliche Elemente alleine im Notentext präsent sind, etwa in Form von Titeln, Widmungen, Anmerkungen etc. In diesen Fällen gilt es, musikalische Bedeutungsträger mit weiteren Inter- bzw. Kontexten sinnvoll zu verbinden. ${ }^{56}$ Freilich kann das auch schnell zu leichtfertigen und wenig plausiblen Deutungen führen. So kritisiert etwa Williams ${ }^{57}$, dass das sog. ıvirág‘-Motiv (benannt nach seinem prominenten Gebrauch in Kurtágs Die Sprüche des Péter Bornemisza für Sopran und Klavier op. 7, 1963/76) von Kurtág-Interpret*innen zur Legitimation von intertextuellen Bezügen angeführt wird, ohne dass der strukturelle und inhaltliche Kontext ausreichend beachtet werde. Im von Williams betrachteten Fall eines Játékok-Stücks z. B. ist das in Frage stehende Motiv nicht aus op. 7, sondern aus der Telefonnummer des Widmungsträgers abgeleitet.

54 Kunkel 2008, 96.

55 Ebd., 151.

56 So gelingt es z.B. Simone Hohmaier (2002), anhand von Hommage-Kompositionen Kurtágs die gemeinsame Verwurzelung verschiedener Aspekte der Musik von Kurtág und Péter Eötvös in den Theorien Erno' Lendvais aufzuzeigen.

57 Williams 2001, 66f. 
Daher kommt der Autor zu dem Schluss: »In this case, as in countless others, there is no stable relationship between musical motive and association or extra-musical idea, only a network of fluid and totally subjective associations. ${ }^{158}$

Die hier beschriebene effektive Auflösung eines möglichen Botschaftsinhalts in polyvalente Bedeutungsfülle und subjektive Sinnassoziation stellt freilich nur das eine von zwei Extremen in Kurtágs Musik dar, gleichwohl neigt eine Vielzahl von Kurtágs Stücken eher diesem als dem entgegengesetzten Pol zu (offensichtliche Sinnstiftung wie im Fall der oben erwähnten musikalisch-mimetischen Bebilderung von Textsemantik). Eine textzentrierte hermeneutische Lesart, welche zwischen den skizzierten Extrempolen fluktuierende bzw. oszillierende Bedeutungen in einem eindeutigen Sinngehalt zu fixieren sucht, stößt daher schnell an ihre Grenzen.

Einen Wink, wie die bislang aufgezeigte Perspektive konstruktiv ergänzt werden könnte, gibt eine Bemerkung Kurtágs, die dem am Anfang dieses Abschnitts angeführten Zitat angefügt ist. Nach der exemplarischen Definition einer Botschaft heißt es dort weiter: „Das ist das Allerwichtigste. Die Briefe der Madame de Sévigné. ${ }^{59}$ Der von Kurtág assoziativ in den Raum gestellte Hinweis auf die berühmte französische Briefschriftstellerin lässt ein signifikantes Identifikationspotenzial vermuten. Und tatsächlich kann man eine gewisse Parallelität erkennen: Genauso wie die Marquise de Sévigné zeit ihres Lebens keinen einzigen ihrer an zahllose Adressat*innen gerichteten Briefe veröffentlichte, dabei aber eine Geschliffenheit des Stils und Akkuratesse der Gedankenführung kultivierte, welche zumindest potenziell an ein breiteres Publikum gerichtet zu sein scheint, so ist auch Kurtágs Musik in ihrer Genese zwar aus persönlich-privaten Kommunikationszusammenhängen motiviert, im Resultat aber so durchdacht und so durchgestaltet, dass sie gleichsam nach einer öffentlichen Hörerschaft ruft. Deshalb (und natürlich auch, weil Kurtág im Gegensatz zur Marquise de Sévigné seine Werke veröffentlicht) muss man davon ausgehen, dass es sich bei der festgestellten Ambivalenz zwischen Privatheit und Öffentlichkeit, Botschaftscharakter und Inhaltshermetik, Sinnsuggestion und Sinnverweigerung nicht um das sozusagen zufällige Ergebnis eines privatistischen Mitteilungsbedürfnisses, sondern um das Mittel einer (mehr oder weniger bewussten) künstlerischen Strategie handelt. In diesem Sinne spricht etwa auch Williams in Hinblick auf die durch Kurtágs Musik aufgerufene Fülle der Assoziationen von Kurtágs »desire to partially reveal these associations in the titles and dedications of his pieces. But along with the suggestion of associations goes a desire also to obscure those associations ${ }^{60}$ Für die analysierenden und interpretierenden Rezipient*innen von Kurtágs Musik heißt das, Kurtágs Kompositionen nicht wie inhaltszentrierte Statements (etwa im Beethoven'schen Sinne), sondern primär als Andeutungs- und Versteckspiele (wie z. B. in Schumanns Musik) zu behandeln. Und das beinhaltet ganz wesentlich, bei der Erschließung der offerierten Botschaft die (bereits weiter oben angedeuteten) medialen und performativen Dimensionen aus verschiedenen Blickwinkeln zu betrachten. Die re-

58 Ebd., 67.

59 Kurtág 2010, 50.

60 Williams 2001, 66. 
sultierende Multiperspektivität kann sich dabei in gewisser Hinsicht auf Kurtágs eigene musikalische Interpretationspraxis berufen. Denn

[t] hose who have heard Kurtág teach, might have noticed, that for him, music lives and becomes meaningful in its infinite associative potential. [...] one can dig out the inner meaning of a musical gesture only if one approaches it from many directions, listening to its multiple associations and connections. ${ }^{61}$

Darstellend-musikalische und analysierend-rezeptive Interpretationen von Kurtágs Musik ließen sich mithin insofern als strukturgleich bezeichnen, als beide einen Sinngehalt von verschiedenen Punkten aus wiederholt einkreisen. Ein Unterschied liegt freilich darin, dass sich in einer musikalischen Aufführung nur jeweils eine Version eines musikalischen Sinngehalts aktualisieren lässt, während das Privileg der analytischen Interpretation darin besteht, auf die potenzielle Vielheit der Deutungsmöglichkeiten aufmerksam machen zu können. ${ }^{62}$ Und da Kurtágs Musik nicht nur den Hinweis auf dieses vielschichtige Bedeutungspotenzial, sondern auch den wiederholten Versuch seiner Aktualisierung geradezu einfordert, könnte man so weit gehen, nicht einen konkreten Sinngehalt, sondern den unabschließbaren Prozess des semiotischen Interpretationsvollzugs selbst zur eigentlichen Botschaft der Musik zu erklären. Marshall McLuhans berühmte These, nach der sich das Medium seinem Inhalt aufprägt, kontrafaktisch abwandelnd ließe sich somit behaupten: The interpretation is the message.

\section{SAMUEL BECKETT: WHAT IS THE WORD OP. 30A/B}

Für die im Folgenden versuchte Skizze einer palimpsestartigen analytischen Interpretationsweise bietet sich Kurtágs Samuel Beckett: Mi is a szó. Siklós István tolmácsolásában Beckett Sámuel üzeni Monyók Ildikóval [Samuel Beckett: What is the Word. Samuel Beckett sendet Wörter durch Ildikó Monyók in der Übersetzung von István Siklós] mit seinen beiden Versionen op. 30a für Stimme und Pianino (1990) sowie op. 30b für Rezitation, Stimmen und im Raum verteiltes Kammerensemble (1991) ${ }^{63}$ vor allem aus zwei Gründen an: Einerseits weist es für die drei durchzuspielenden Perspektiven zahlreiche und offenkundige Ansatzpunkte auf, andererseits ist es in der Kurtág-Literatur bereits wiederholt untersucht worden. Letzterer Umstand bietet den Vorteil, dass die betrachteten Aspekte auf existierenden Untersuchungsergebnissen aufbauen bzw. mit diesen ergänzt und verglichen werden können; der Überblick über die bisher gewählten Interpretationsansätze lässt zudem Rückschlüsse über die implizite These zu, dass Kurtágs Musik besonders offen für die Erschließung durch unterschiedliche Zugänge ist.

61 Frigyesi 2002, 401.

62 Und vielleicht liegt hierin auch der Grund für die effektive Deutungsdominanz von Kurtágs eigenen Interpretationsauffassungen; denn im Laufe einer Einstudierung gilt es notwendigerweise, sich an einem gewissen Punkt festzulegen; und wenn sich eine Entscheidung als stimmig erwiesen hat, wird sie naturgemäß nicht immer wieder von neuem infrage gestellt werden.

63 Wenn im Folgenden Aussagen getroffen werden, die gleichermaßen für op. 30a wie op. 30b gelten, so wird von sop. 30 « die Rede sein, ansonsten werden beide Versionen getrennt angesprochen. 
Die drei hier gewählten Interpretationsperspektiven basieren auf drei Paradigmen, die die Musikologie der letzten Jahrzehnte in besonderem Maße beeinflusst haben: (Inter-) Textualität, Medialität und Performativität. Die Gliederung des folgenden Kapitels durch diese drei Paradigmen wird weder in jedem Detail strikt durchgehalten noch ist sie als methodisch modellhaft für Kurtág-Analysen allgemein zu verstehen. Vielmehr soll die leitende These der möglichen Überschichtung interpretativer Ebenen formal verdeutlicht und damit auch inhaltlich plausibel gemacht werden.

\section{(Inter-)Textualität}

\section{Text als Intertext}

Als Intertexte im Sinne der Intertextualitätsklassifikation Gérard Genettes ${ }^{64}$ sind im Fall von Kurtágs op. 30 zum einen die sprachlichen Textvorlagen anzusehen: Samuel Becketts What is the Word bzw. dessen durch István Siklós vorgenommene Übertragung ins Ungarische Mi is a szó; zum anderen muss auch das kurz vor Ende prominent erscheinende Zitat aus Béla Bartóks Zweitem Violinkonzert (1937/38) dazu gezählt werden. Im Gegensatz zu vielen anderen Kompositionen Kurtágs handelt es sich in beiden Fällen um ganz offensichtliche Intertexte: die sprachlichen Textvorlagen sind lückenlos vertont (die Siklós-Übersetzung in op. 30a, zusätzlich das Original Becketts in der Chorpartie von op. 30b). Das Bartók-Zitat ist zwar leicht abgewandelt übernommen, im Notentext wird aber explizit auf seinen Autor hingewiesen (an entsprechender Stelle heißt es »arioso, ommagio a Bartók«).

Jenseits der, für sich genommen, relativ aussageleeren Klassifikation kann die intertextuelle Perspektive vor allem dabei helfen, die Beziehungen der beiden Intertexte zum Haupttext, dem Notentext, analytisch zu erhellen. Was das Verhältnis von Textvorlage und Notentext angeht, hat Michael Kunkel in der bislang umfangreichsten Untersuchung von op. 30 eine grundlegende und insgesamt profunde Arbeit vorgelegt. ${ }^{65}$ Sie zeigt, dass der Beckett-Text insofern im konventionellen Sinne vertont wird, als er als strukturelle und inhaltliche Vorlage dient, die in erkennbare Beziehung zur musikalischen Ebene der ihn deklamierenden Monodie tritt. Die Konkretion und mimetische Qualität dieser Beziehung kann dabei stark variieren; sie reicht von direkter textueller Korrelation bis zu nicht oder nur assoziativ zu erschließenden Bezügen. In Takt 32/33 (Bsp. 1) wird beispielsweise das «mi « des Textes als notentextuelle Solmisationssilbe in direkter Weise als Ton $e^{1}$ übersetzt, während die prominente repetitio der Silbe »hol« [»wo«] auf dem $d$ in Takt 38 (Bsp. 2) sich in eher assoziativer Weise als eine Art letzter Suchbewegung deuten ließe, bevor in Takt 40 mit der Terz $a^{2}-c i s^{3}$ ein neues Element eingeführt wird. Die zuletzt genannte losere Form der Bezugnahme ist freilich mitbedingt durch den radikal reduzierten semantischen und bildlichen Gehalt von Becketts Text. Als dessen einzige Bedeutungskonstante wird die titelgebende Frage »What is the word? « durch

64 Vgl. Genette 1993.

65 Vgl. Kunkel 2008, 68-134. 


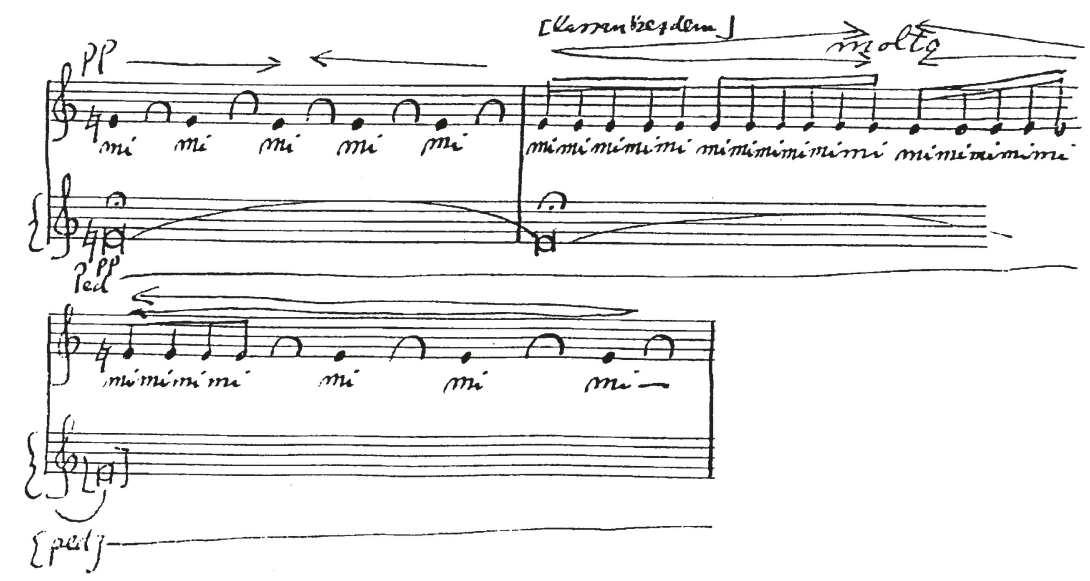

Beispiel 1: György Kurtág, Samuel Beckett: What is the Word op. 30a, T. 32/33; oberes System: Stimme, unteres System: Pianino (C 2006 EMB Music Publishers)

ständige Umkreisung und kontextuell variierte Wiederholung exponiert; die übrigen immer wieder neu zusammen- und umgestellten Wortkonstellationen etablieren hingegen keine stabilen Sinngefüge, sie verbleiben gleichsam in einer vorsyntaktischen selbstreferenziellen Schleife. Wenn man diesen beiden Arten der textlichen Bedeutungskonstitution formale Prinzipien zuordnet, dann entscheidet sich Kurtág in der großformalen Gestaltung seiner Vertonung gegen das Permutationsprinzip der Wortschleifen und für das Variationsprinzip der Fragewiederholung. Dabei wählt er eine Art expressive Variationsform, die - im Rahmen kurzer, meistens ein oder zwei Takte umfassender Abschnitte - in immer neuen Formen von Deklamationsarten und Ausdruckscharakteren den Text artikuliert. In der intervallisch-diastematischen Binnenstrukturierung der Monodie kommt hingegen auch das Permutationsprinzip zum Tragen. So wird im Laufe des Stücks in immer wieder neuen Spielarten das in den ersten sieben Takten exponierte Reservoir von intervallischen und melodischen Charakteristika ausgeschöpft (unterschiedliche chromatische Klein- und vor allem Großterz-Ausfüllungen, Quint- bzw. Quart-/TritonusKombinationen, vorherrschend absteigende Diastematik).

Aus der Abfolge der aneinandergereihten Abschnitte ergibt sich eine klar erkennbare Dramaturgie der einsätzigen, ca. zwölf- bis 17-minütigen ${ }^{66}$ Komposition (von Kunkel ${ }^{67}$ sinngemäß beschrieben als >Materialexposition - Exposition von Deklamationstypen Verdichtung - Verunsicherung und Reprise - Ruhepunkt - Zusammenbruch ‘). Das oben erwähnte Bartók-Zitat (Bsp.3), welches auf dem Hauptthema des langsamen zweiten Satzes von Bartóks Zweitem Violinkonzert beruht (Bsp. 4), fungiert dabei gleichermaßen als Ruhe- wie als Wendepunkt, was mit verschiedenen von Kurtág vorgenommenen

66 Aus der individuellen Interpretation von Kurtágs relativer Dauern- und Pausennotation können erhebliche Unterschiede der Gesamtlänge resultieren, als Orientierung dienen hier Minimal- und Maximalwerte der erhältlichen Aufnahmen von op. 30a (vgl. dazu weiter unten).

67 Kunkel 2008, 77-85. 


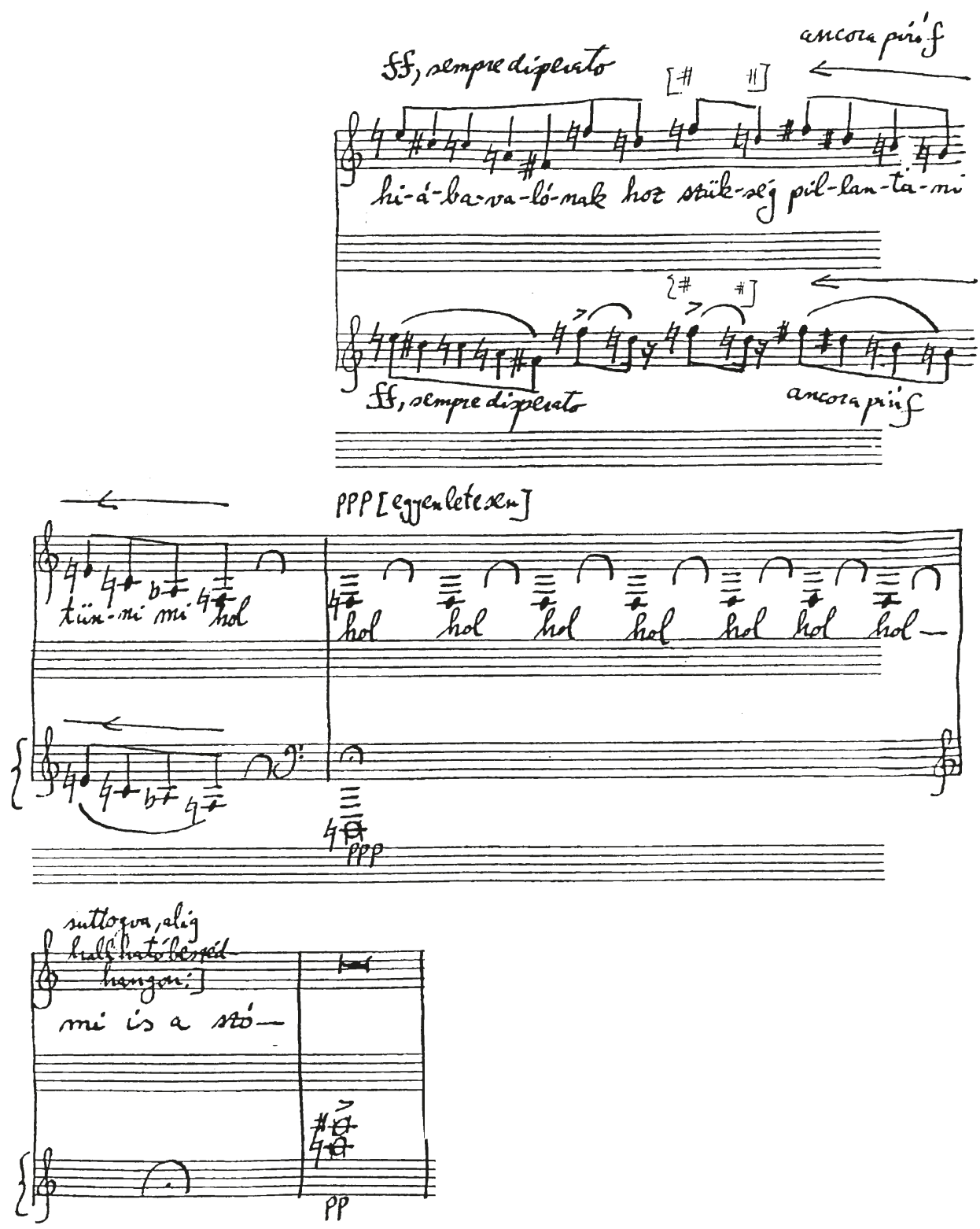

Beispiel 2: György Kurtág, Samuel Beckett: What is the Word op. 30a, T. 37-40, oberes System: Stimme, unteres System: Pianino (ㄷ 2006 EMB Music Publishers) 


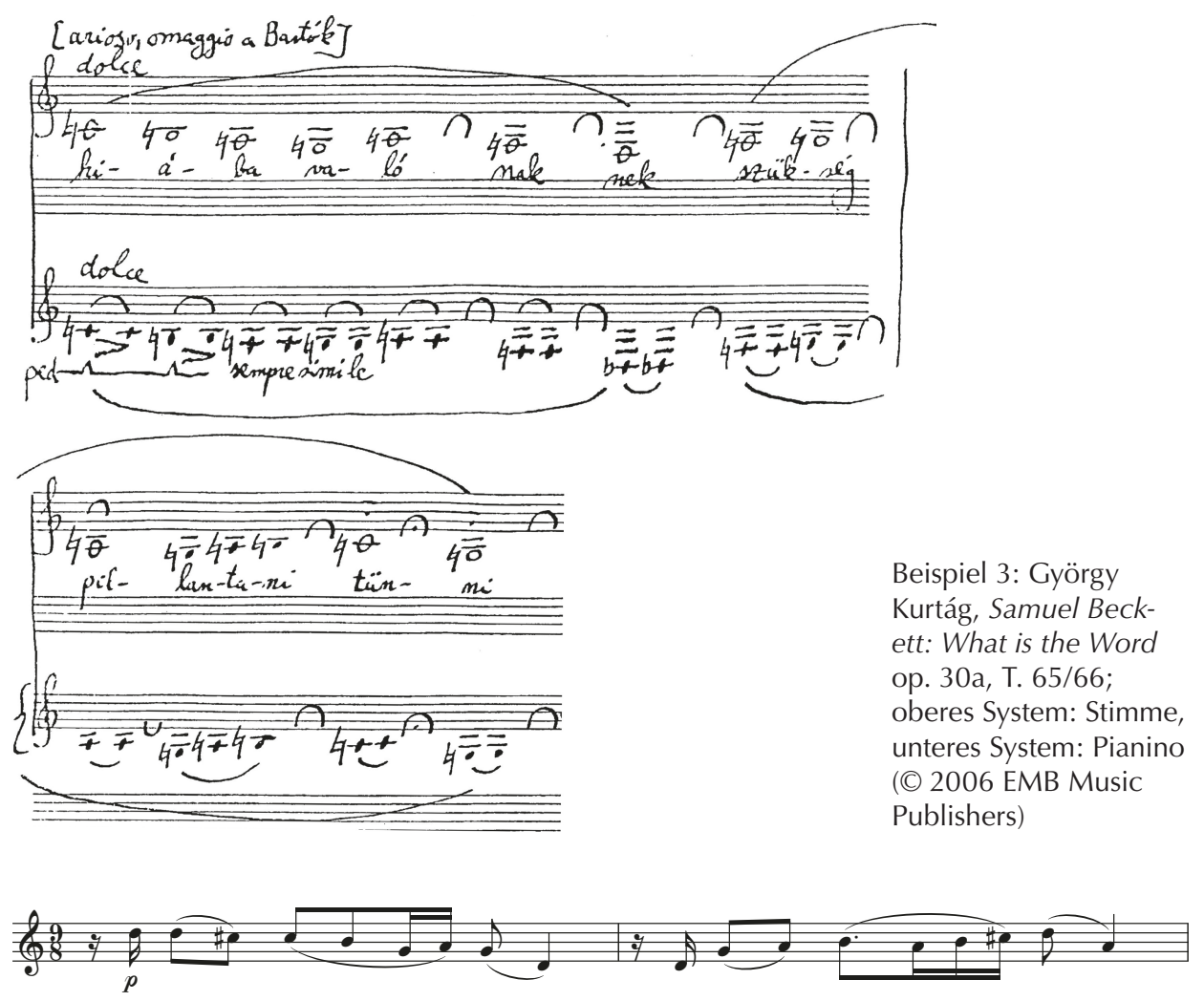

Beispiel 4: Béla Bartók, Violinkonzert Nr. 2, 2. Satz, T. 2/3, Solovioline (C) 1946 Hawkes \& Son London)

Transformationen zu tun hat. So wird das Thema, während es bei Bartók - nach einem tonalitätsabsteckenden Einleitungstakt - direkt am Satzanfang steht und als thematische Keimzelle des gesamten Satzes dient, in What is the Word erst nach ca. drei Vierteln des Stücks eingeführt und zudem erkennbar als Fremdkörper inszeniert (durch den vorhergehenden Takt, der auf einem repetierten Klavierton $h$ innehält und gleichzeitig leittönig den ersten Zitat-Ton vorbereitet, sowie durch die in dieser Form einmalig auftretenden Tonwiederholungen der Klavierstimme). Zusätzlich wird die bei Bartók klar erkennbare symmetrische Struktur des Themas aufgebrochen: die Symmetrie der Syntax (Phrase und Gegenphrase als Vordersatz eines thematischen Fortspinnungstypus bei Bartók) durch die unregelmäßige Durchsetzung mit Pausen; die Symmetrie der Dauern durch die relative Dauern- und Pausennotation im langsamen Tempo sowie durch die (ungefähre) Dauernverdoppelung der beiden bei Bartók in der ersten Phrase auftretenden Sechzehntel; und schließlich die Symmetrie der Diastematik durch die Transposition des tiefsten Melodietones um einen Halbton aufwärts (des statt c), sodass das ssymmetrisch-perfekte، melodische Rahmenintervall der Oktave zur großen Septime gestaucht wird (bei dem an dieser Stelle im Manuskript stehenden $d$ der Stimme handelt es sich offensichtlich um 
einen Fehler $\left.{ }^{68}\right)$. Diese strukturellen Veränderungen bewirken, dass das Thema zwar als Bartók-Zitat erkennbar bleibt, dabei allerdings von einem Entwicklungsnukleus zu einem Ruhepol umgedeutet wird. Seine diatonisch-tonale Charakteristik wird im Anschluss aber auch aufgegriffen - durch die Weiterführung in einem Fis-Dur-Dreiklang (T. 67), der dann - nach kurzer Aufwärtsbewegung zur Quinte - in die chromatische Abwärtsbewegung (T. 69-70) überleitet, die den langen, gedehnten Schlusstakten vorhergeht.

Betrachtet man die Aussagen der Kurtág-Literatur zu dem Bartók-Zitat, so fällt auf, dass diese über die dezente, aber - wie gesehen - nicht insignifikante textuelle Transformation des Zitats weitgehend hinwegsehen und stattdessen vor allem die Bedeutung der Person und Musik Bartóks für Kurtág im Allgemeinen in den Fokus rücken ${ }^{69}$; allein Simone Hohmaier weist - durch ihre intertextuelle Methodik sensibilisiert, aufgrund eines anderen Untersuchungskontextes allerdings nur en passant - darauf hin, dass die vorgenommenen Veränderungen strukturell motiviert sind, da der Tonraum des Bartók-Zitats an verschiedenen Stellen vorbereitend erschlossen wird. So gedeutet wird die chromatische Auffüllung des Großterzraums $g$ - $h$ in den Takten 1 bis 4 (Bsp. 5) genauso als strukturelle Vorbereitung erkennbar wie die diatonische Exposition von (reinen und übermäßigen) Quartintervallen in den Takten 22 bis 24 (Bsp. 6). Zudem greift die Transposition des $c$ zum des im Verhältnis zum umliegenden $f$ außerdem das zuvor an zahlreichen Stellen prominent eingesetzte Intervall der großen Terz auf. Diese aneignende Einpassung in die diastematische und strukturelle Gesamtlogik macht klar, dass das Zitat nicht alleine auf das Signifikat des intertextuellen Verweises zielt, sondern dass die textuell-materielle Qualität (es handelt sich ja um ein explizites Notenzitat), also der Signifikant des Zitats von ebenso großer Bedeutung ist. Im Kontext einer semantischen Interpretation würde das der textbezogenen Deutung Kunkels, der op. 30a als Scheitern einer versuchten Sprachfindung interpretiert, einen relativierenden Aspekt hinzufügen: Solange die - so Kurtág über Bartóks Musik - »Muttersprache « ${ }^{70}$ nicht vollkommen verloren ist, kann ihre umformende Integration vor vollkommenem Sprachverlust bewahren.

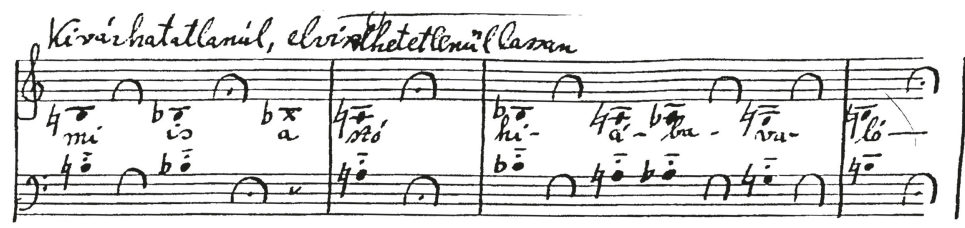

Beispiel 5: György Kurtág, Samuel Beckett: What is the Word op. 30a, T. 1-4, oberes System: Stimme, unteres System: Pianino (C 2006 EMB Music Publishers)

68 In der Partitur von op. 30b ist des notiert und in allen Aufnahmen (jenen von op. 30b sowie auch in dem später zitierten Workshop-Ausschnitt, in dem Kurtág selbst op. 30a einstudiert) wird des gesungen.

69 Beispielhaft die ansonsten sehr detailreich argumentierende Untersuchung Kunkels (2008, 88-92).

70 Vgl. Hohmaier 1997, 13. 

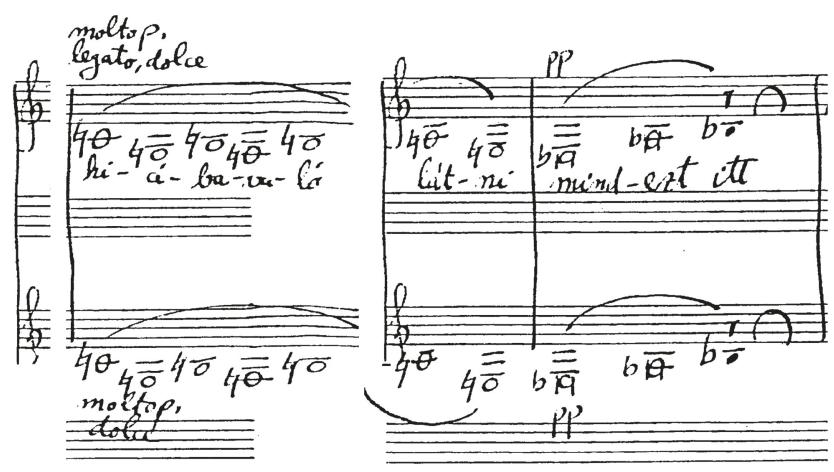

Beispiel 6: György Kurtág, Samuel Beckett: What is the Word op. 30a, T. 22-24, oberes System: Stimme, unteres System: Pianino (C) 2006 EMB Music Publishers)

\section{Op. 30a und op. 30b als Hypertexte}

Die beiden Versionen von op. 30 stehen in einem komplexen Verhältnis zueinander. Das beginnt bereits bei der Werkgenese. So suggerieren neben der Opuszählung vor allem die Unterschiede in Umfang und Differenziertheit des Notentexts (op. 30a: zehnseitige Partitur für Stimme und Klavier; op. 30b: 44-seitige Partitur mit zusätzlichem Ensemble und Chor) das sequenzielle Verhältnis von Nukleus (op. 30a) und erweiternder Ausarbeitung (op. 30b). Der Umstand, dass Kurtág op. 30b nur ca. ein Jahr nach der Niederschrift von op. 30a und in - für seine Verhältnisse - relativ kurzer Zeit ${ }^{71}$, zudem offensichtlich auf Grundlage von Particell-Eintragungen in der Klavierversion komponierte ${ }^{72}$, legt aber nahe, dass bei der Komposition von op. 30a die spätere Version zumindest konzeptionell schon mitgedacht war. Doch auch wenn man die Entstehungsgeschichte ausblendet und das Verhältnis beider Versionen - nach Genette - als hypertextuelles beschreibt, so ist nicht eindeutig zu bestimmen, welche der Versionen als Hypotext (Vorlagentext) bzw. Hypertext (abgeleiteter Text) zu gelten hat. So ist zwar offenkundig, dass op. 30b den Notentext von op. 30a erweitert, indem verschiedene Ebenen hinzugefügt werden: eine instrumentatorische durch das große Ensemble und den Chor, eine raummusikalische durch die Verteilung der Akteure im Aufführungsraum, eine textuelle durch die Integration von Becketts durch den Chor artikulierten Originaltext sowie eine formale durch die Hinzufügung eines instrumentalen Prologs und Epilogs. Entgegen der Interpretation Kunkels, der die Expansion von op. 30b als "Verdeutlichung " ${ }^{73}$ der kompositorischen Aussage von op. 30a ansieht, könnte man aber mit ähnlichem Recht, mit dem man op. 30b als Elaboration von op. 30a begreifen kann, auch op. 30a als Komprimierung von op. 30b auffassen. Begründbar ist das durch die strukturelle Kompatibilität beider Versionen. So gibt es - sieht man vom hinzugefügten Prolog und Epilog ab - in op. 30b keine einzige Note, die nicht in eindeutiger Weise mit dem Tonsatz von op. 30a korreliert, und auch Prolog und Epilog sind mit dem Text von op. 30a strukturell verbunden: der Prolog als ausinstrumentierter Cluster des für die Monodie konstruktiven Großterzintervalls und der

71 Vgl. Kunkel 2008, $98 f$.

72 Vgl. Zenck 2002, 418.

73 Kunkel 2008, 98. 
Epilog durch den impliziten Bartók-Bezug, der - wie Hohmaier bemerkt ${ }^{74}-$ sich aus der permutierten Zitation der im ersten Satz von Bartóks Zweitem Violinkonzert zu findenden vierteltönigen Umspielungsfigur ergibt (Bsp. 7: Bartóks Original, Bsp. 8: der Anfang des Epilogs).

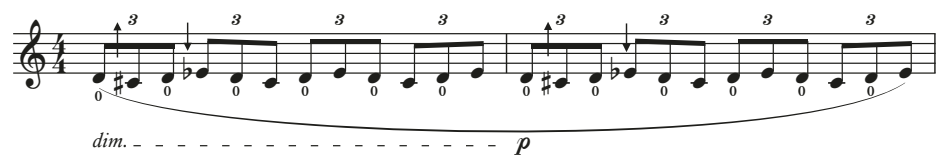

Beispiel 7: Béla Bartók, Violinkonzert Nr. 2, 1. Satz, T. 303/304, Solovioline (C 1946 Hawkes \& Son London)

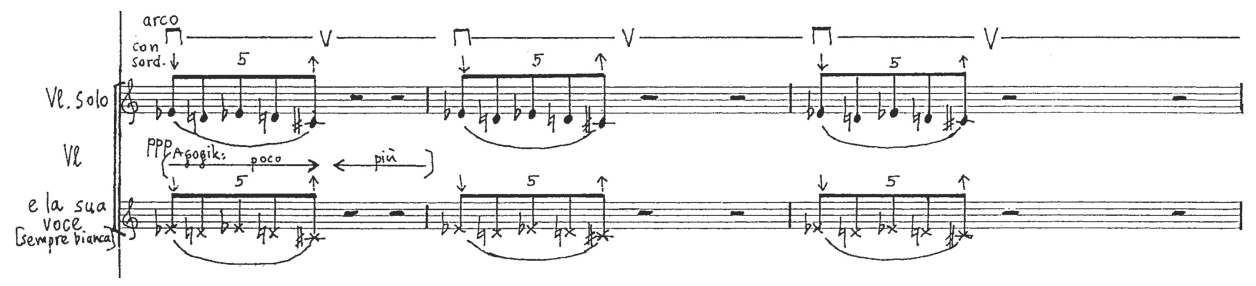

Beispiel 8: György Kurtág, Samuel Beckett: What is the Word op. 30b, Epilog: Studienziffer a, Violine (@ 1991 Editio Musica Budapest)

Die Frage, ob op. 30b als Entfaltung (in konventionellerer Terminologie: als erweiternde Instrumentierung) von op. 30a oder ob umgekehrt op. 30a als Einfaltung von op. 30b (im Sinne eines Klavierauszugsubstrats) anzusehen ist, hat freilich auch Konsequenzen für eine inhaltliche Interpretation. So lassen sich für die durch Kunkels Rede von »Verdeutlichung insinuierte These, op. 30b würde den Bedeutungsgehalt von op. 30a präzisieren und konkretisieren, im Notentext von op. 30b sowohl unterstützende als auch widersprechende Momente finden. Z. B. werden Kontraste in op. 30b in der Tat sehr viel deutlicher konturiert (etwa die große Terz $\mathrm{a}^{2}-\mathrm{cis}^{3}$ in Takt 39 von op. 30a, die bei Ziffer nn in op. 30b als leuchtende, sich von der düster-trauermarschartigen Umgebung deutlich abhebende Klangfarbe inszeniert ist), gleichzeitig werden aber auch Ambivalenzen intensiviert: sei es in Details wie die in op. 30b bei Taktziffer g zu findende ffluktuierender Instrumentation des in op. 30a durch eine Halteklang des Klavier dargestellten Tritonus g-cis ${ }^{1}$ (Bsp. 9), oder sei es in der allgemeinen Tendenz, dass aus der - oft schon dynamisch signifikanten, teils auch wesentlich durch den Chor mitgetragenen - Verstärkung der aufeinander folgenden Deklamationscharaktere die in op. 30a deutlich erkennbare Klarheit der übergeordneten Dramaturgie zugunsten von Binnenkontrasten abgeschwächt wird. In keinem Fall zuzustimmen ist einer These von Judit Frigyesi: Wenn sie schreibt, die "polyphony of contrasting emotions« von op. $30 \mathrm{~b}$ thematisiere »no longer merely the anxiety of expression« wie in op. 30a, sondern weitergehend »the problem 
of existence ${ }^{75}$, impliziert sie damit die teleologische Erhöhung des expressiven Gehalts und mithin den Primat von op. 30b, welcher durch einen textuell-vergleichenden Blick schwer zu rechtfertigen ist. Zwar lässt sich in Bezug auf op. 30b in der Tat eine größere Vielschichtigkeit und Binnenkomplexität der textuellen Ebenen konstatieren, im Resultat entsteht dadurch aber - wie bereits angedeutet - nicht automatisch ein höheres Maß an Beziehungsreichtum, vielmehr halten sich die Effekte von struktureller Konkretion und Abstraktion, von Verdeutlichung und Verunklarung des expressiven Gehalts in etwa die Waage. Sowohl Frigyesis als auch Kunkels Deutungen müssen unter der hier eingenommenen Perspektive also tendenziell als idiosynkratische Interpretationen gelten, welche der eigentümlichen Konstellation, dass sich die Versionen von op. 30a und op. 30b in einem engen, annähernd austauschbaren hypertextuellen Verhältnis zueinander befinden, nicht gerecht werden.

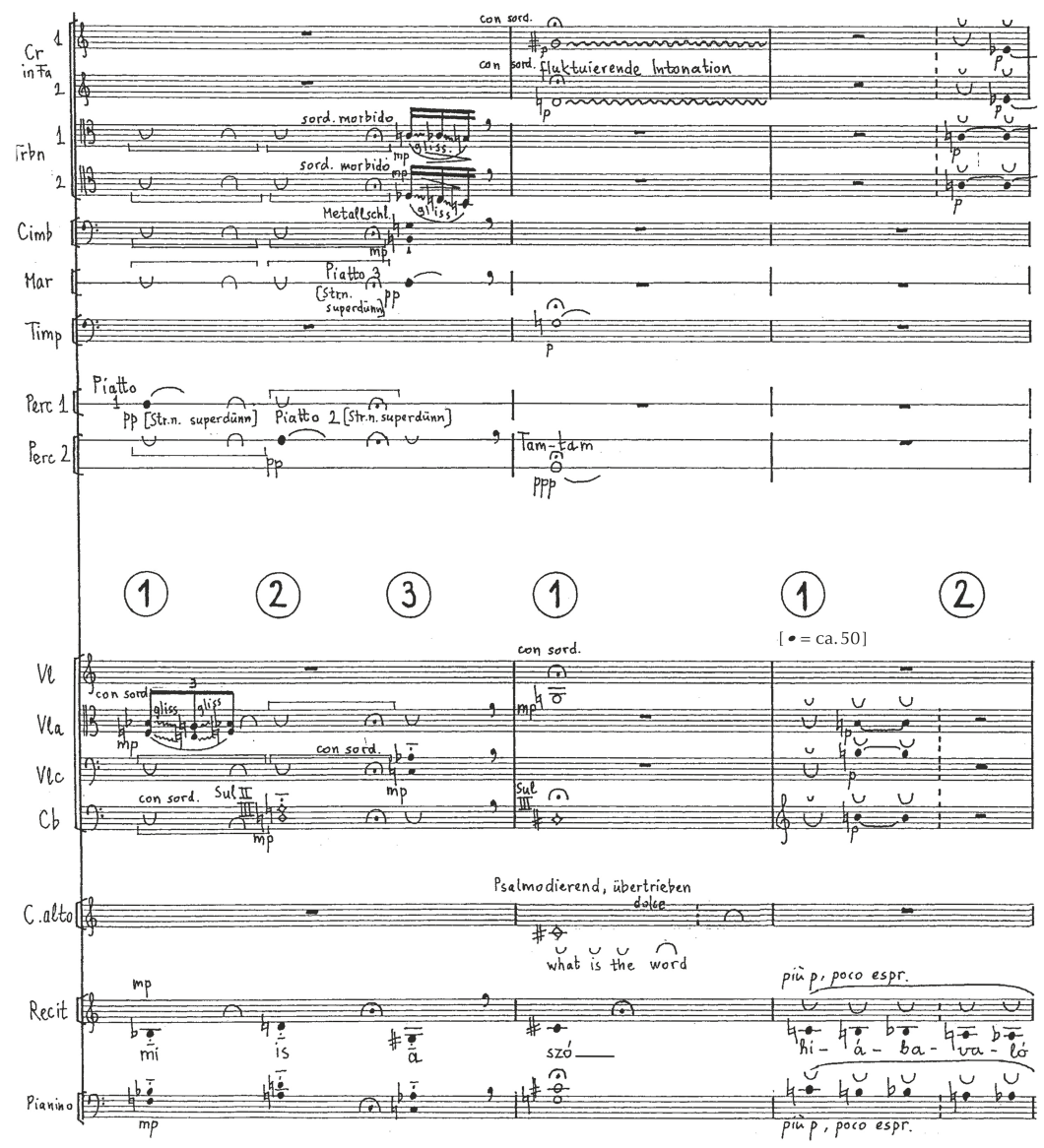

Beispiel 9: György Kurtág, Samuel Beckett: What is the Word op. 30b,

Partiturausschnitt, Takt g, h, I (C 1991 Editio Musica Budapest)

75 Frigyesi 2002, 398. 


\section{Das Klavier als Textgenerator}

Die im letzten Abschnitt versuchte hypertextuelle Beschreibung des Verhältnisses von op. 30a und op. 30b konturiert nicht nur grundlegende Unterschiede und Gemeinsamkeiten beider Versionen, sie lässt auch Rückschlüsse über die Bedeutung der beiden Hauptakteure von op. 30a zu. In Hinsicht auf die Funktion des Klaviers ist dabei jener Umstand entscheidend, der als textuelle Kompatibilität beider Versionen angesprochen worden ist. Denn als gemeinsamer Nenner der Korrelation des Notentexts von op. 30a und op. 30b kann der Tonsatz des Klaviers gelten. Er doppelt die Monodie der Gesangstimme in op. 30a und kann zugleich als Gerüst des gesamten Tonsatzes von op. 30b angesehen werden - besonders an jenen Stellen, welche die innerhalb des meist lineareinstimmigen Klaviersatzes äußerst selten zu findenden harmonischen Implikationen ausformulieren (z.B. die Klangfläche nach Ziffer s). Als gleichsam innerer Nukleus des gesamten Werkkomplexes kann das Klavier daher - textuell-funktional formuliert - als ‘Generator des Notentextes angesehen werden. Denn auch die Gesangstimme, welche zwar ohne Frage eine hohe phänomenale und performative Präsenz besitzt, ist strukturell gesehen eher aus dem Klavier-Tonsatz abgeleitet als umgekehrt. Deutlich wird das z. B. in Takt 44 (Bsp. 10), wenn das Klavier eine markante Geste, nämlich eine vom $a^{2}$ ausgehende und auf diesen Ton wieder zurückführende Wellenbewegung spielt, welche von der Singstimme nicht in ihrer Gesamtheit und partiell oktavversetzt gedoppelt wird. Aus textueller Perspektive wäre die durch Kunkels Rede vom Klavier als »)Krückeく der Monodie ${ }^{76}$ implizierte These, dass dem Klavier eine untergeordnete, rein instrumentelle, bei der Sprachfindung unterstützende Rolle zukomme, daher zu relativieren.
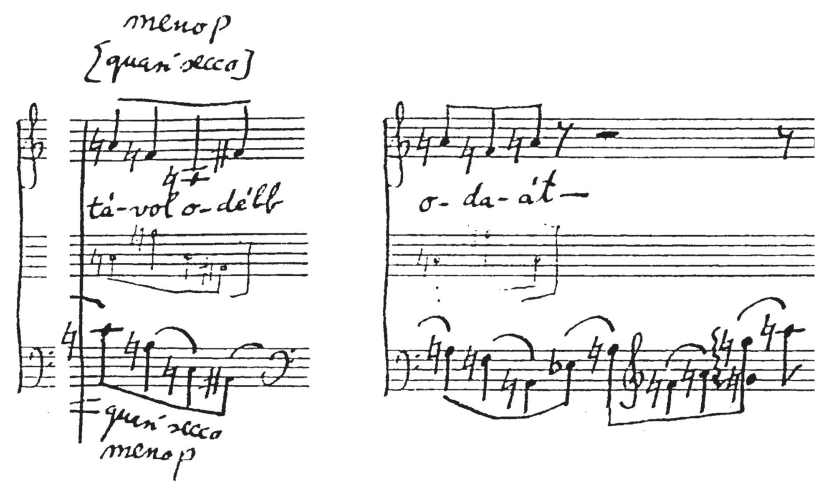

Beispiel 10: György Kurtág, Samuel Beckett: What is the Word op. 30a, T. 44, oberes System: Stimme, unteres System: Pianino (C) 1991 Editio Musica Budapest)

\section{Die Sängerin als Exekutorin des Notentexts}

Aus dem Notentext geht eine nähere Spezifizierung oder Charakterisierung der Gesangstimme nicht bzw. nur sehr rudimentär hervor. Zwar wird im Werktitel mit »[...] sendet Wörter durch Ildikó Monyók [...] « die Person der ungarischen Sängerin, für die und auf deren Anregung hin Kurtág das Stück komponierte, explizit erwähnt; in Besetzungsan-

76 Kunkel 2008, 77. 
gabe oder Notentext selbst finden sich allerdings - assoziative Andeutungen ausgenommen, die mit Monyóks musikalischem Hintergrund in der populären und Schlagermusik in Verbindung gebracht werden können (»in modo popolare «77 in Takt 14 von op. 30a) - keine konkreten Hinweise darauf, dass das Stück für eine spezifische Ausführende konzipiert ist; eine von der konventionellen Funktion einer musikalischen Interpretin abweichende Sonderrolle wird rein textuell mithin nicht signalisiert. Technizistisch formuliert könnte man die Rolle der Interpretin daher als jene einer 'Exekutorin des Notentextes bezeichnen. Die bewusste Überspitzung dieser Formulierung darf dabei freilich nicht als positive Thesenbildung verstanden werden; nicht nur kontextuell, in Hinblick auf die bereits mehrfach erwähnte Interpretationsaffinität und -sensibilität von Kurtágs Schaffen, wäre das abwegig, auch notentextuell würden die zahlreichen Vortragsbezeichnungen von op. 30 eine solche Sichtweise relativieren, da ihre zumeist expressive Qualität einer neutralen `Exekutions-Haltung ‘ widerspricht. Mit der Einführung des Exekutionsbegriffs soll vielmehr ex negativo darauf aufmerksam gemacht werden, dass - mehr als bei allen anderen bisher betrachteten Aspekten - der Bedeutung der Sängerin für op. 30 durch eine rein notentextuelle Betrachtungsweise nicht annähernd beizukommen ist, sondern dass sich stattdessen - und noch deutlicher als bei anderen `Botschafts-Kompositionen Kurtágs - die Person Ildikó Monyók als gleichermaßen Text-Adressatin wie -Ausführende als eine im Notentext nicht bzw. nur paratextuell bezeichnete Bedeutungsschicht in die Komposition mit einschreibt.

\section{Medialität}

\section{Text als Medium}

Im Vergleich zur (inter-)textuellen Position verlagert eine medientheoretisch ausgerichtete Textinterpretation den Schwerpunkt von der Identifikation eines semantisch-referenziellen Sinngehalts hin zu den Beschaffenheiten der bedingenden Mitteilungsverhältnisse sowie den involvierten materiellen und - legt man ein an personifizierten Figuren orientiertes Medienkonzept ${ }^{78}$ zugrunde - personellen Instanzen. Der Betrachtung von Becketts Text What is the Word, der seinen Sinn weniger aus den nur noch residual vorhandenen denotativen Bedeutungen generiert, als dass er mit exemplifizierenden Mitteln - den kreisenden Textbewegungen seiner permutierten Textformationen - Sprache auf ihre Mitteilungsfunktion hin befragt, kommt ein solcher Ansatz naturgemäß entgegen. Aber auch bei der Untersuchung von Kurtágs Vertonung lässt ein medialer Fokus wichtige Aspekte deutlicher hervortreten. So wird schon im bereits oben zitierten ausführlichen und überaus ungewöhnlichen Titel von op. 30 eine multiple Auffächerung der

77 Diese Vortragsbezeichnung findet sich auch in anderen Kurtág-Werken, dort wird sie aber in der Regel ausschließlich als Hinweis auf eine svolksmusikalische` Vortragsart gebraucht. Im Fall von op. 30 ist durch den Bezug auf die Person Monyóks auch eine Deutung als spopuläre Musikı (im Sinne etwa von Pop-, Schlagermusik) möglich.

78 Wie beispielsweise das in Krämer 2008 konturierte. 
medialen Mitteilungsstruktur suggeriert, durch die der Autor Kurtág hinter eine Kette von medialen Instanzen zurücktritt: Der Autor Beckett als primäre Senderinstanz lässt demnach mithilfe des Übersetzers Siklós durch die Interpretin Monyók eine Botschaft (implizit: an den/die Hörer*in als Empfänger*in) ausrichten. An der Konstruktion dieser - kommunikationstheoretisch gesprochen - Signalweiterleitungskette ist auffällig, dass alleine personelle Instanzen genannt werden und die Übertragungsmedien, derer diese sich bedienen, ausgespart bleiben (der Text bei Beckett, die Stimme bzw. Bühnenpräsenz bei Monyók und natürlich die musikalische Komposition beim nicht erwähnten Gesamtautor Kurtág). Diese personalisierte Lesart des behaupteten Nachrichten- oder Botschaftscharakters von op. 30 macht vor allem eines deutlich: Im Gegensatz zu einer Medientheorie, welche die Eigendynamik und Eigengesetzlichkeit von (vor allem technischen) Medien betont (so etwa die Position der Toronto Schoo/79), geht es Kurtág um die im Mediengeschehen involvierten menschlichen Akteur*innen (Sender, Überbringer*in, Adressat*innen, Empfänger*innen). Es sind ganz wesentlich die jeweiligen Möglichkeiten und Eigenheiten dieser Akteur*innen, die den Verlauf des Kommunikationsprozesses prägen. Bezogen auf die hermeneutische Erschließung des »medialen Sinns « ${ }^{80}$ eines Spiels - in diesem Fall: des Interpretationsspiels von op. 30 - lässt sich daraus die Notwendigkeit ableiten, eine textuelle Perspektive durch eine medienanalytische Komponente zu ergänzen. Denn wenn jene gleichsam die Struktur der Möglichkeit medialer Kommunikation aufzudecken hilft, so ist die medienfokussierte Perspektive in der Lage, die Verfasstheit der involvierten Medien wie auch ihren Gebrauch durch die am Kommunikationsprozess beteiligten Akteur*innen genauer zu verstehen.

\section{Op. 30a und op. 30b als Kommunikationssituationen}

Wenn man sich unter skizzierter Perspektive dem Vergleich der medialen Anlage beider Versionen von op. 30 zuwendet, dann fällt als erstes die extrem unterschiedliche Rahmensetzung der jeweiligen Kommunikationssituation auf: Während die minimale Besetzung von op. 30a eine Aufführung in einem intimen kammermusikalischen Kontext suggeriert, verlangt die Realisierung von op. 30b allein schon aufgrund der Anzahl der beteiligten Musiker und ihrer räumlichen Verteilung auf verschiedene Gruppen (die Instrumentalist*innen um das Publikum herum, der Chor in diesem selbst positioniert) einen großen Konzertsaal (das Vorwort nennt beispielhaft den Kammermusiksaal der Berliner Philharmonie, den Wiener Mozart-Saal und den großen Saal der Budapester Musikakademie). Allein diese unterschiedlichen Aufführungssituationen resultieren in einem fundamental differenten Kommunikationscharakter der beiden Versionen: Der fasst man ihn als strukturellen Gerüstsatz auf - im Kern identische Notentext, welcher im Fall von op. 30a die Grundlage einer scheinbar aus der Nähe beobachtbaren und erfahrbaren, gleichsam privaten kommunikativen Interaktion zwischen Interpreten und Publikum dient, gewinnt durch die mediale Rahmensetzung von op. 30b eine distanzier-

79 Vgl. Hartmann 2000, 239.

80 Gadamer 1960, 109. 
te, mehrfach vermittelte ${ }^{81}$ und damit sozusagen öffentliche Qualität. Mit dieser Differenz lässt sich auch das oben beschriebene Phänomen der intensivierten Kontrastbildung von op. 30b in Verbindung bringen: Damit die verkündete Botschaft überhaupt Gehör finden kann, muss sie verstärkt werden. Statt der von Kunkel gewählten Rede von »Verdeutlichung" trifft der Begriff der `Amplifikation` (nicht zuletzt aufgrund seiner medial-technischen Konnotation) das Verhältnis von op. 30b zu op. 30a daher sehr viel besser.

\section{Das Klavier als Klangmedium}

Führt man die zuvor aufgestellte These vom Klavier als dem funktionalen Protagonisten von op. 30 aus medientheoretischer Perspektive weiter, so wird man auf die mediale Funktion des Klavierklangs aufmerksam. Diese lässt sich in op. 30a am besten am Verhältnis von pianistisch-instrumentaler und vokaler Klanglichkeit entfalten. Martin Zenck charakterisiert dieses Verhältnis, wenn er die Rolle des Klaviers beschreibt als »accompaniment, which probably does not really serve as a support for the intonation, but especially as a deepening and colouring of each sound $\aleph^{82}$. Der in dem Zitat implizit enthaltenen Kritik an Kunkels »Krücken«-These (das Klavier als Stütze des Gesangs) ist dabei genauso zuzustimmen wie der grundsätzlichen Charakterisierung der klanglichen Beziehung von Gesang und Klavier als »colouring" der monodischen Linie. Gleichwohl ist zu hinterfragen, ob das klangliche Verhältnis tatsächlich als hierarchisches Subordinationsverhältnis von vokaler Hauptstimme und instrumentaler Begleitung, von stimmlichem Grundton und klavierklanglicher Einfärbung aufzufassen ist. Denn für genau das Gegenteil spricht nicht nur, dass der Tonsatz des Klaviers extrem einfach gehalten ist, sondern auch, dass dessen Artikulation nur sehr begrenzt ausdifferenziert wird. Das Potenzial der ohnehin schon relativ unflexiblen Klanglichkeit des Instruments wird also nicht ansatzweise ausgelotet, während die unterschiedlichen Vortragsbezeichnungen in der Gesangspartie eine variable Stimmgebung und somit nuancenreiche Klangfarbe suggerieren. Die strukturelle Bezogenheit der Stimme auf das Klavier in op. 30a würde sich nach dieser Sichtweise also auch klanglich-medial fortsetzen.

Im Vergleich dazu stellt sich in op. 30b das klangliche Verhältnis von Klavier und Stimme sowohl vielschichtiger als auch einfacher, weniger ambivalent dar. So bleibt die aus op. 30a bekannte Binnenrelation von Klavier und Stimme zwar erhalten, durch die Hinzufügung des umfangreichen Instrumentalapparates tritt die Beziehung von Klavierund Ensembleklang aber prominenter hervor (hinzu kommt, dass die solistische Stimme nun sehr viel stärker mit der vokalen Klangfarbe des Chors korrespondiert). Weil der instrumentale Tonsatz - wie oben erwähnt - gänzlich aus dem pianistischen Klavierpart abgeleitet ist, liegt hier nun aber ganz eindeutig der Fall einer klangfarblichen Ausinstrumentierung vor. Und obwohl im mitunter entstehenden Palimpsest der instrumentalen

81 Zur medialen Entfaltung des Klavierklangs in instrumentale Klangfarben sowie den akustischen Raum kommt außerdem noch eine weitere performative Vermittlungsebene: Die Geige tritt an verschiedenen Stellen des Stücks quasi solistisch zu den beiden Hauptakteuren (Sängerin und Pianist*in) hinzu und wird besonders im instrumentalen 'Sospirando-Epilog` gleichsam zur medialen Stellvertreter-Stimme.

82 Zenck 2002, 417. 
Klangfarben das Klavier (wohlgemerkt handelt es sich auch im großbesetzten Kontext um ein Pianino) zeitweise fast ausgelöscht wird (etwa im Cluster des Beginns), so bleibt das grundlegende Verhältnis zwischen relativ starrem Klavierklang und seiner teils sehr farbenreichen Instrumentalumhüllung stets präsent. ${ }^{83}$ Auch in op. 30b fungiert das Klavier also gleichsam als medialer Fokus des klanglichen Geschehens.

Wenn das Klavier - in op. 30b noch deutlicher als in op. 30a - primär als Medium von Klang in Erscheinung tritt, so ist die Stimme aus medientheoretischer Sicht vor allem als Artikulationsmedium des Beckett-Textes anzusehen. Neben der daraus erwachsenden semantischen Dimension wird mithin auch die Stimmträgerin, die Sängerin, fokussiert, kurz: Die Sängerin erscheint als Textbotschafterin. Automatisch wird dadurch auch die im Stücktitel angesprochene Personifikation der Sängerin relevant und kontextuelles Wissen über die Person Ildikó Monyók zur beachtenswerten Interpretationsquelle. Bereits erwähnt wurde, dass die Anregung für die Komposition von op. 30a von Seiten Monyóks ausging. Eine auslösende Rolle spielte dabei der persönliche Hintergrund, dass Monyók nach einem Autounfall unter einer akuten Sprechstörung litt, wodurch sie auch daran gehindert wurde, ihre Karriere als populäre Schauspielerin und Sängerin in der Unterhaltungsmusik weiterzuführen. ${ }^{84}$ Mit dieser Zusatzinformation gewinnt die im Titel angesprochene mediale Vermittlungsdimension der Komposition eine ganz neue Bedeutungstiefe, denn das Stück kann als eine vollgültige Botschaft im weiter oben beschriebenen Sinne Kurtágs aufgefasst werden: Sie erhält daraus ihren Sinn, dass Ildikó Monyók sich sauf den Weg machts, es zu interpretieren, und das heißt auch ganz praktisch: in einem mühsamen Prozess daran arbeitet, ihre Sprechprobleme zu überwinden. Hier gilt also in ganz konkreter Weise die oben aufgestellte These: Nicht die (gleichermaßen rezeptive wie performative) Fixierung eines (textuell decodierbaren) Sinnes, sondern der Prozess der Interpretation selbst ist die Botschaft.

\section{Performativität}

\section{Der Text als Spielvorlage}

Durch eine performative Betrachtungsweise, die Notentexte als Anweisungen für musikbezogene Handlungen ansieht, wird im Fall von Kurtágs op. 30 ein Aspekt in den Mittelpunkt gerückt, der schon in Becketts Textvorlage angelegt ist bzw. bei Beckett allgemein eine wichtige Rolle einnimmt: der Spielcharakter des Geschehens. Die Beckett und Kurtág verbindende Auffassung, dass ein Spiel sich nicht als Gegenteil von Ernst bestimmt, sondern vielmehr eine (wie es im Játékok-Vorwort heißt:) »todernste« oder, man könnte auch sagen, existenzielle Dimension besitzt, beruht auf einer charakteristischen

83 Unterstützt wird das einerseits durch die - klangliche Transparenz fördernde - Raumverteilung der Instrumentalgruppen und andererseits durch die zentrale - durch visuelle auch klangliche Prominenz suggerierende - Positionierung des Klaviers auf dem frontalen Podium.

84 Vgl. Kunkel 2008, S. $68 f$. 
Janusköpfigkeit, die in Theorien des Spiels immer wieder angesprochen wird. ${ }^{85}$ So ist ein Spiel einerseits als ein Geschehen definiert, in das von den Spielern aktiv eingegriffen werden kann, andererseits weist das Spiel aber auch eine Eigengesetzlichkeit auf, welche die Spielenden in eine passive Dynamik zwingt; kurz: die Spieler spielen das Spiel und gleichzeitig spielt das Spiel die Spieler. ${ }^{86}$ Wenn man nun diese beiden gleichermaßen kontradiktorischen wie - für das Gesamtkonzept - komplementären Aspekte von Spiel (die im Übrigen in jeder performativen musikalischen Interpretation naturgemäß präsent sind) auf den Notentext von What is the Word bezieht, dann fällt auf, dass sie in beiden Versionen jeweils unterschiedlich nuanciert sind. So könnte man davon sprechen, dass der Spielraum bzw. das Spielfeld von op. 30b in verschiedener Hinsicht abgesteckter, determinierter ist als bei op. 30a. Das hängt schon mit der großen Besetzung zusammen, deren koordinierteres Zusammenspiel neben einem/einer Dirigent*in (gleichsam als Spielleiter*in) auch eine genauere notationelle Bestimmung erfordert. So enthält die Partitur von op. 30b zusätzliche, den Zeitfluss strukturierende Zeichentypen (einerseits metrische Taktbestimmungen und andererseits Einsätze für die Stellen, in denen Kurtág seine spezifische relative Dauernnotation verwendet ${ }^{87}$ ). Op. 30a lässt dagegen nicht nur in zeitlicher Hinsicht größere Freiheiten zu, auch die Gestaltung der zu erschließenden sowie durch die (zum Teil assoziativen) Spielanweisungen suggerierten musikalischen Ausdrucks- und Deklamationscharaktere sind weniger klar umrissen (die Spielanweisungen der Solo-Stimme sind in op. 30b zwar identisch, durch die oft sehr detailliert vorgeschriebenen Ausdruckstypen der die Stimme immer wieder doppelnden Chorpartien werden sie allerdings indirekt mit definiert).

Wenn man nun also die Notentexte von What is the Word als Vorlagen eines freieren (op. 30a) bzw. organisierteren Spiels (op. 30b) begreift, so darf das freilich nicht zu dem (bereits bei Frigyesi beobachteten, dort anders begründeten, aber im Resultat identischen) interpretativen Fehlschluss verleiten, op. 30b sei in seiner Wirkung oder in seinem Gehalt weniger sverspielt‘, mithin sexistenzieller als op. 30a. Denn die Beurteilung solcher oder ähnlicher Qualitäten kann nicht allein aufgrund des Notentexts erfolgen (welcher, es sei noch einmal betont, nicht das Spiel selbst, sondern die Spielvorlage darstellt); vielmehr gilt es, in ganz wesentlichem Maße dessen Aktualisierung (die Bedingungen seiner Aktualisierung wie auch tatsächlich erfolgte Aktualisierungen) mit einzubeziehen.

85 Vgl. Gadamer 1960, 107-116.

86 Die vielleicht besten Beispiele in Becketts CEuvre dafür sind das berühmte Endgame (1956), dessen Text Kurtág als Vorlage eines immer wieder in Angriff genommenen und seit einigen Jahren intensiv verfolgten Opernprojekts dient, sowie das weniger bekannte Play (1962/63). Die in den Titeln enthaltene begriffliche Differenzierung des Englischen korrespondiert im Übrigen tendenziell mit den beiden erwähnten Spielaspekten ('games: passiv, splayı: aktiv).

87 Die Funktion der Dauernnotation, eine intuitive, ıgefühlte`Zeitgestaltung zu suggerieren, wird dadurch de facto ausgelöscht, denn durch die von dem/der Dirigent*in gegebenen Einsätze entscheiden die Musiker*innen nicht mehr selbst über die zeitliche Proportionierung, sondern reagieren nur noch auf die vorgegebene Dauernstruktur. 


\section{Op. 30a und op. 30b als Aufführungssituationen}

Wenn man die unter medialer Perspektive bereits deutlich markierte Differenz der jeweiligen Kommunikationssituationen von op. 30a und 30b aus performance-theoretischer Sicht weiter konturiert, so könnte man die getroffene Unterscheidung in die Kategorien sprivat (op. 30a) und ı̈ffentlich` (op. 30b) mit zwei verschiedenen Spielmodi und Aufführungstypen in Verbindung bringen: Der private Aufführungscharakter von op. 30a wäre demnach jenem Spielmodus, der in der Performance-Theorie als performative Handlung oder kurz: als sPerformen beschrieben wird ${ }^{88}$, sowie dem Konzert als konventionellem Aufführungstyp zuzuordnen; die öffentliche Aufführungssituation von op. 30b hingegen ließe sich mit dem Spielmodus des Darstellens und dem Aufführungstyp sMusiktheater assoziieren, denn obwohl das Stück ausdrücklich für die Konzert- und nicht etwa die Opernbühne konzipiert ist, weist es schon in seiner Disposition auffällige stheatrale`Momente auf: die räumliche Verteilung der Instrumente oder auch die explizite Bezeichnung der Bühne im Vorwort als »scene».

Freilich sind diese Verknüpfungen als tendenzielle Zuordnungen innerhalb bipolarer Felder zu verstehen; für eine Interpretation können sie gleichwohl aufschlussreich sein, um die performative Komponente von op. 30 differenzierter zu charakterisieren. So spricht beispielsweise Zenck bereits im Titel seines Beitrags von »Theatricality of a Non-theatrical Music « ${ }^{89}$, also von einer theatralen Qualität einer im genretechnischen Sinne nicht-szenischen Musik. Op. 30 und andere Stücke Kurtágs enthalten demnach theatrale Implikationen, welche von sich aus aber keine szenische Umsetzung verlangen, »because their theatrical imagination and simulation surpasses any dramatic reality «. ${ }^{90}$ Bezeichnenderweise unterscheidet der Autor allerdings nicht zwischen op. 30a und op. 30b, obwohl er ein Element, das eindeutig für eine größere musiktheatralisch-szenische Gewichtung von op. 30b spricht, explizit anführt: den Epilog, »Sinfonia [epilogo scenico] « überschrieben, dessen Kernelement eine mikrotonale Seufzer-Circulatio um $d^{1}$ ist, die von dem/der solistischen Violinist*in - mit ostentativer Bogenführung visuell sowie mit hohem Bogendruck akustisch markiert - gespielt und dabei mitgesummt wird. Neben diesem ganz offensichtlichen Moment musiktheatralen Darstellens tragen zur ‘Bespielung، der großen Bühne von op. 30b aber auch bereits erwähnte Faktoren bei: die Amplifikation der Monodie durch Chor- und Instrumentalapparat sowie die raummusikalische Disposition (inklusive des theatralen Effekts, der sich aus der visuellen und akustischen Durchdringung von Chor- und Publikumsraum ergibt).

Als weiteres Argument für die hier getroffene Zuordnung lässt sich die unterschiedliche Aufführungsgeschichte der beiden Version von op. 30 anführen. Während op. 30b wiederholt und inzwischen auch von verschiedenen Sängerinnen in großem Rahmen aufgeführt und daher auch in der Öffentlichkeit relativ breitenwirksam rezipiert worden ist, fanden die wenigen Aufführungen von op. 30a in einem intimen, beinahe hausmusikalischen Rahmen und - zu Zeiten ihres aktiven Bühnenlebens - ausschließlich mit Ildikó Monyók als Sängerin statt. Zudem erfolgte die Uraufführung, obwohl das Stück

88 Vgl. Fischer-Lichte 2012, 41-44.

89 Zenck 2002, 411.

90 Ebd., 420. 
ein Jahr vor op. 30b fertiggestellt war, zwei Jahre nach der Premiere von op. 30b und im Gegensatz zu drei Einspielungen von op. 30b ist von ihm auch keine Aufnahme erhältlich. Kurze Ausschnitte einer Interpretation sind gleichwohl in einer Passage von Judit Keles Film L'homme allumette / Der Streichholzmann zu sehen, die einen gemeinsamen Workshop von Monyók und Kurtág dokumentiert (Videobsp. 1).

1)) http://storage.gmth.de/zgmth/media/889/Poller_Kurtag_Video01.mp4

Videobeispiel 1: Ildikó Monyók und György Kurtág bei einer Probe zu György Kurtág, Samuel Beckett: What is the Word op. 30a für Stimme und Pianino (1990)

(Judit Kele, L'homme allumette / Der Streichholzmann: Kurtág-Portrait, ZDF/Arte 1996, 45:34-48:16)

Auch in diesen säußeren` Umständen also spiegelt sich der im Kern ganz unterschiedliche performative Charakter der beiden Versionen wider: Während in op. 30a eine private Botschaft gleichsam unmittelbar sperformt « wird, inszeniert op. 30b eine Botschaft in einem theateraffinen Spiel von Musik-Darsteller*innen.

\section{Das Klavier als Autorpersonifikation}

Die These von der Rolle des Klaviers als strukturelles wie klangliches Zentrum von op. 30 lässt sich in performativer Hinsicht ausbauen, wenn man zwei Aspekte berücksichtigt: So gilt es zum einen zu bedenken, dass das Klavier jenes Instrument ist, welches Kurtág den Zugang zu eigener wie fremder Musik ganz wesentlich ermöglicht und - wie oben erwähnt - im Kompositionsprozess eine eminent wichtige Rolle spielt; ein in diesem Kontext wichtiges Detail ist die explizite Besetzung des Pianino in op. 30, denn das Stehklavier ist für Kurtág ein Instrument, das er vor allem aufgrund seines - in vielen seiner Werke verlangten - intimen `Super-Sordino «-Klangs besonders schätzt..$^{91}$ Bemerkenswert ist zum anderen, dass Kurtág offensichtlich darauf Wert legte, bei der Uraufführung von op. 30b sowie ebenfalls bei Aufführungen von op. 30a selbst den Klavierpart zu übernehmen. Auch die Konzeption des sehr einfachen Klaviersatzes, der fast durchgängig mit nur einer Hand zu bewältigen ist, kann laut Kurtág92 auf seine eigenen pianistischen Fähigkeiten zurückgeführt werden. Zusammengenommen mit den bereits eingeführten Beschreibungen der Klavierfunktion (`Textgenerator`, 'Klangmedium`) ließe sich das Klavier daher in gewisser Weise als Personifikation des Komponisten begreifen; denn in seiner generativ-strukturell zentralen, gleichwohl nie als solcher prominent exponierten Rolle vertritt es die Autorfunktion und spiegelt gleichermaßen das KomponistenSelbstverständnis Kurtágs treffend wider. Die Selbstbesetzung Kurtágs als pianistischen Stückinterpreten könnte aber auch noch weitergehend als versteckte autopoietisch-performative Botschaft gelesen werden. Durch sie würde die oben beschriebene Geste des Komponisten, hinter die übermittelnden Instanzen der suggerierten Botschaft zurückzu-

91 Kele 1996, 45:26-45:31.

92 Ebd., 45:05-45:16. 
treten, in der Personalunion von Klavier und Spieler auf ihren ansonsten verborgenen Ausgangspunkt zurückgeführt.

\section{Die Sängerin als Rolle}

Die besondere Stellung der Gesangspartie lässt sich erst aus performativer Perspektive angemessen erschließen. Denn die im Titel von op. 30 vollzogene Einschreibung Ildikó Monyóks als Überbringerin einer Botschaft entfaltet den vollen Umfang ihres Sinns erst dann, wenn sie in Konstellation mit der Sängerin Monyók als Person und ihren performativen Fähigkeiten bzw. Einschränkungen gelesen wird. So wird die von Becketts Text im literarischen Raum symbolisch verhandelte Sprachfindungsthematik durch die Verknüpfung mit dem individuellen Schicksal Monyóks in die Sphäre aktualisierter Realität geholt. Die permutierte Vertonung der fragmentierten Sprache Becketts in der zögernden, mit Pausen durchsetzten, zuweilen abbrechenden und dann in anderen Deklamationstypen wieder neu anlaufenden Monodie gewinnt durch den Bezug auf eine reale Sprachstörung, auf ein tatsächliches Stottern, das Potenzial, zu etwas anderem, jenseits von Textsignifikant und Objektsignifikat liegendem Dritten zu werden. Diese Verwandlung bedarf freilich der performativen Aktualisierung. Im Fall des auf den Modus des Performens angelegten op. 30a scheint dies in performativ schlüssiger Weise nicht anders als durch Ildikó Monyók selbst möglich gewesen zu sein (die Sängerin starb 2012); nur die Person, die gewissermaßen selbst zu einem Teil der Komposition geworden ist, kann diese im eigentlichen Sinne performen, denn nur sie ist in der Lage, die bei darstellenden Aufführungssituationen immer schon vorhandene Differenz zwischen Spielanweisung und Spielgeschehen alleine in ihrem So-Sein, in ihrer Präsenz zumindest annähernd ${ }^{93}$ aufzuheben.

Op. 30b hingegen ist durch seine implizit theatrale Dimension von vornherein auf Darstellung angelegt. Auch die Gesangspartie muss daher zumindest in ihrem konzeptionellen Kern als eine Rolle angesehen werden - selbst in dem Fall (wie z. B. bei der Uraufführung), dass Monyók selbst die Gesangspartie übernahm; denn dass Monyók gewissermaßen sich selbst spielen konnte, liegt durchaus im Bereich des Möglichen (und das kann auch Kunkel entgegen gehalten werden, der es als »Problematik« von op. 30b ansieht, dass »Monyóks Gebrechen in zweifelhafter Weise gross ausgestellt wird « ${ }^{94}$, während doch nicht notwendigerweise Monyóks Gebrechen, sondern mitunter auch die Repräsentation von Monyóks Gebrechen ausgestellt werden könnte). Andere Sängerinnen hingegen sind in jedem Fall - in besonderem Maße bei op. 30a, aber eingeschränkter auch in op. 30b - damit konfrontiert, dass sie in ihrer individuellen Interpretation sozu-

93 Dass diese Differenz nie ganz überwunden werden kann, weil eine Bühnensituation einen nicht vollkommen auzulöschenden Rest von repräsentierendem Darstellungscharakter behält, deutet folgende Aussage Kurtágs an, die sich auf Monyóks Erarbeitung des Werkes bezieht: "She did this very hard working to be herself here on the stage.« (Kele 1996, 48:18-48:23)

94 Kunkel 2008, 133. Im Fall von op. 30a wären Kunkels Bedenken gegen die Ausstellung von Monyóks Gebrechen dagegen unmittelbar nachzuvollziehen. Und - wie ein Ausschnitt aus dem Workshop mit Kurtág zeigt (vgl. Kele 1996, 46:30-46:57) - scheint Monyók in der intimen Zusammenarbeit mit Kurtág in der Tat besonders affizierbar und mithin exponierbar zu sein. 
sagen immer auch ein wenig Monyók spielen müssen oder - anders formuliert - dass sie jenen Teil von Monyóks Beitrag zur Darstellung bringen müssen, der den Sinnzusammenhang zwischen Komposition und Klangresultat gewährleistet.

Betrachtet man in dieser Hinsicht die drei erhältlichen Einspielungen von op. 30b zwei davon mit Ildikó Monyók (Monyók/Claudio Abbado 1996; Monyók/Péter Eötvös 1994), eine mit Piroska Molnár (Molnár/Beat Furrer 2010) -, so kann man eine interessante Polarität feststellen, die sowohl mit dem Aufnahmezeitpunkt als auch mit der jeweiligen Interpretin des Stimmparts zusammenhängt. So kann man generell konstatieren, dass sich Monyóks Interpretation der Vokalpartie durch eine größere Freiheit gegenüber dem Notentext auszeichnet, und das insbesondere an Stellen, die bereits durch expressive Spielvorgaben eine hohe Ausdrucksintensität suggerieren. So wird z. B. die »sempre disperato « vorzutragende absteigende Melodielinie von Takt kk (die ersten beiden Takte von Bsp. 2 zeigen die analogen Takte in op. 30a) von Monyók in unrein krächzender, fast schreiender Stimme und zudem mit ausgeprägter Agogik interpretiert (Audiobsp. 1 und 2). Dieselbe Stelle in der Aufnahme mit Molnár und Furrer (Audiobsp. 3) wird hingegen fast exakt im vorgeschriebenen Tempo (Achtel = 100) und ohne größere Schwankungen bzw. erst am Ende mit einem Rallentando ausgeführt, und zudem wirkt die genau intonierte, ssachlichere` Gesangsart Molnárs deutlich weniger affektiv aufgeladen.

(1)) http://storage.gmth.de/zgmth/media/889/Poller_Kurtag_Audio01.mp3

Audiobeispiel 1: György Kurtág, Samuel Beckett: What is the Word op. 30b, auf: Hommage à Andrei Tarkovsky, Deutsche Grammophon 437840, 1996, Sopran: Ildikó Monyók; Arnold Schönberg Chor, Ensemble Anton Webern, Mus. Leitung: Claudio Abbado, 4:56-5:05

(1)) http://storage.gmth.de/zgmth/media/889/Poller_Kurtag_Audio02.mp3

Audiobeispiel 2: György Kurtág, Samuel Beckett: What is the Word op. 30b, auf: György Kurtág Portrait Salzburg 1993, Col Legno 31870, 1994, Sopran: Ildikó Monyók; Tomkins Vokalensemble, Budapest Festival Orchestra, Mus. Leitung: Péter Eötvös, 6:30-6:41

(2)) http://storage.gmth.de/zgmth/media/889/Poller_Kurtag_Audio03.mp3

Audiobeispiel 3: György Kurtág, Samuel Beckett: What is the Word op. 30b, auf: Salzburg Biennale. Festival for New Music 2009, Neos 10947, 2010, Alt: Piroska Molnár; Vokalensemble Zürich, Ensemble Contrechamps, Mus. Leitung: Beat Furrer, 6:46-6:59

Was hier beispielhaft für die Gesamtinterpretation zu beobachten ist - die genaue Beachtung der im Notentext fixierten Hauptparameter (Tonhöhen, Tempo, Dauernproportionen) bei Molnár/Furrer gegenüber einer freieren, affektiv-emotionalen Vortragsweise bei Monyók - kann leicht mit den bisher untersuchten Aspekten in Zusammenhang gebracht werden. Während für Monyók die notierte Vokalstimme eher als Orientierung gebende Vorlage für das dient, was sie idealerweise in der Übereinstimmung von darstellender Persona und dargestellter Person zum Ausdruck zu bringen versucht, erfolgt die interpretative Erschließung bei Molnár/Furrer primär als Darstellung des Notentexts. 
Dass dabei durchaus auch Lizenzen möglich sind, zeigt eine Stelle, an der Molnár - auffälligerweise ganz ähnlich wie Monyók - einen expressiven, aus hohem Register langsam abwärts glissandierenden Schrei ausstößt (Audiobsp.4-6), obwohl in der Partitur nur ein unbestimmtes Abwärts-Glissando vom relativ tiefen $e s^{1}$ notiert ist (Bsp. 11). Ob sich Molnár dabei an der Interpretation Monyóks orientiert hat, ob sie also hier im oben beschriebenen Sinne Monyóks Rolle tatsächlich bewusst sspielt‘, kann nur gemutmaßt werden, scheint aber, besonders weil ansonsten keinerlei Vortragsbezeichnung die gewählte Vortragsart stützen kann, nicht vollkommen unplausibel.

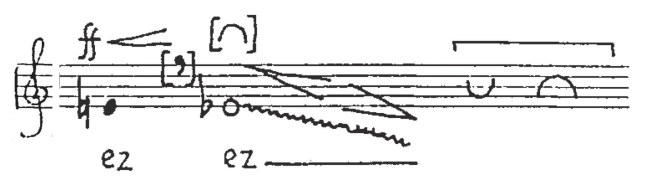

Beispiel 11: György Kurtág, Samuel Beckett: What is the Word op. 30b, Rezitationsstimme, Takt s (C) 1991 Editio Musica Budapest)

(2)) http://storage.gmth.de/zgmth/media/889/Poller_Kurtag_Audio04.mp3

Audiobeispiel 4: György Kurtág: Samuel Beckett: What is the Word op. 30b, auf: Hommage à Andrei Tarkovsky, Deutsche Grammophon 437840, 1996, Sopran: Ildikó Monyók; Arnold Schönberg Chor, Ensemble Anton Webern, Mus. Leitung: Claudio Abbado, 2:23-2:29 http://storage.gmth.de/zgmth/media/889/Poller_Kurtag_Audio05.mp3

Audiobeispiel 5: György Kurtág: Samuel Beckett: What is the Word op. 30b, auf: György Kurtág Portrait Salzburg 1993, Col Legno 31870, 1994, Sopran: Ildikó Monyók; Tomkins Vokalensemble, Budapest Festival Orchestra, Mus. Leitung: Péter Eötvös, 3:15-3:25

(1)) http://storage.gmth.de/zgmth/media/889/Poller_Kurtag_Audio06.mp3

Audiobeispiel 6: György Kurtág: Samuel Beckett: What is the Word op. 30b, auf: Salzburg Biennale. Festival for New Music 2009, Neos 10947, 2010, Alt: Piroska Molnár; Vokalensemble Zürich, Ensemble Contrechamps, Mus. Leitung: Beat Furrer, 3:19-3:27

Eine weitere interessante Beobachtung ergibt sich aus dem Vergleich der beiden MonyókEinspielungen (Bsp. 12, Audiobsp. 7 und 8). Generell fällt auf, dass die Aufnahme mit Abbado deutlich schnellere Tempi sowie kürzere Pausengestaltungen aufweist (Gesamtdauer von ca. zwölf Minuten gegenüber ca. 16 Minuten bei Eötvös), zudem wirkt der bereits angesprochene grundlegende affektiv-expressive Vortragsstil Monyóks in dieser Version noch zugespitzter als in der zwei Jahre später entstandenen Einspielung mit Eötvös - beispielhaft dafür können die Takte k und I stehen (Bsp. 12 zeigt die entsprechende Stelle in op. 30a). Fast scheint es so, als wenn Monyók in der früheren Aufnahme, die in eine Zeit fällt, als die erste Version von What is the Word (op. 30a) noch ganz frisch war, eine ähnliche sexpressiv-ungefilterte` Interpretationshaltung einnimmt wie in der intimen Duo-Konstellation von Vokalpart und Pianino, während sie sich in der zweiten Aufnahme stärker im Modus des Darstellens als nur im Modus des Performens befindet. In jedem Fall kann festgehalten werden, dass auch Monyók, obwohl sie als Adressatin wie 
ausführende Überbringerin einen privilegierten Zugang zur `Botschaftı der Komposition zu haben scheint, Interpretationsweisen wählt, die das Potenzial des Zusammenspiels von fixierter Vorgabe der Partitur und medialer wie performativer Rollendeutung der Interpretin unterschiedlich aktualisieren.

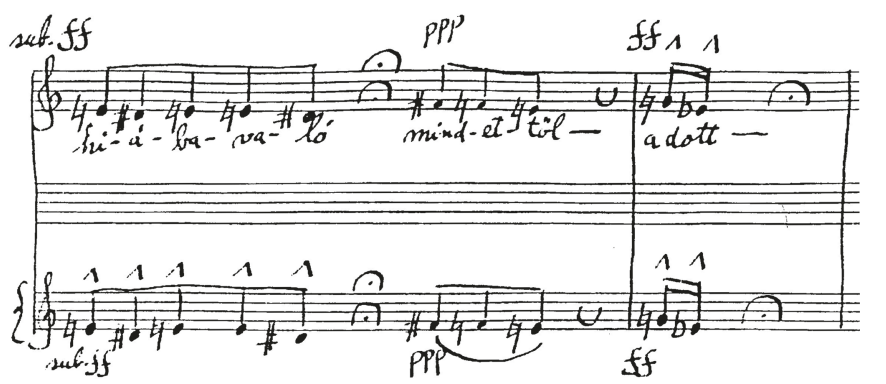

Beispiel 12: György Kurtág, Samuel Beckett: What is the Word op. 30a, T. 11/12, oberes System: Stimme, unteres System: Pianino (C) 2006 EMB Music Publishers)

(2)) http://storage.gmth.de/zgmth/media/889/Poller_Kurtag_Audio07.mp3

Audiobeispiel 7: György Kurtág, Samuel Beckett: What is the Word op. 30b, auf: Hommage à Andrei Tarkovsky, Deutsche Grammophon 437840, 1996, Sopran: Ildikó Monyók; Arnold Schoenberg Chor, Ensemble Anton Webern, Mus. Leitung: Claudio Abbado, 1:22-1:32

(1)) http://storage.gmth.de/zgmth/media/889/Poller_Kurtag_Audio08.mp3

Audiobeispiel 8: György Kurtág, Samuel Beckett: What is the Word op. 30b, auf: György Kurtág Portrait Salzburg 1993, Col Legno 31870, 1994, Sopran: Ildikó Monyók; Tomkins Vokalensemble, Budapest Festival Orchestra, Mus. Leitung: Péter Eötvös, 1:57-2:09

Die Variabilität dieses Verhältnisses spiegelt letztlich eine semiotische Grundkonstellation wider, die für op. 30 allgemein Gültigkeit beanspruchen kann. Denn genauso wie die Rolle der Sängerin genau im Verhältnis von Notentext, vermittelnder Position und interpretierender Darstellung einen zusammenwirkenden Sinnzusammenhalt stiftet, so ist auch die Botschaft von What is the Word weder alleine in der titelgebenden Frage noch alleine in deren Beantwortung zu suchen: »The message is not in the question and not even in the answer, but in the relation between question and answer. ${ }^{95}$

Der Beziehungsgarant zwischen Frage und Antwort, zwischen Zeichen und Objekt heißt in der Terminologie Peirce'scher Semiotik Interpretant. Diese abstrakte Zeichenfunktion in eine konkrete, gleichermaßen individuelle wie intersubjektiv nachvollziehbare Interpretation zu überführen ist die Aufgabe aller Interpret*innen - sei es mit den musikalischen Mitteln darstellender Interpretation oder sei es mit den analytischen Mitteln musikologischer Hermeneutik. 


\section{Literatur}

Adorno, Theodor W. (1956/78), »Fragment über Musik und Sprache« [1956], in: ders., Quasi una fantasia. Musikalische Schriften II (= Gesammelte Schriften, Bd. 16, 249540), hg. von Rolf Tiedemann, Frankfurt a.M.: Suhrkamp 1978, 251-256.

BBVA Foundation (2015), »Interview with György Kurtág«, https://youtu.be/F2p_ R2m67Ys (6.4.2017)

Bleek, Tobias (2010), Musikalische Intertextualität als Schaffensprinzip: Eine Studie zu György Kurtágs Streichquartett »Officium breve« op. 28, Saarbrücken: Pfau.

Cook, Nicholas (2013), Beyond the Score: Music as Performance, New York: Oxford University Press.

Csengery, Adrienne / István Balázs (1986), »Porträt eines Komponisten, aus der Sicht einer Sängerin. Gespräch mit Adrienne Csengery über Persönlichkeit und Kunst von György Kurtág«, in: György Kurtág, hg. von Friedrich Spangenmacher, Bonn: Boosey \& Hawkes, 53-64.

Danuser, Hermann (1992), „Einleitung in ımusikalische Interpretation«", in: ders., Gesammelte Vorträge und Aufsätze, Bd. 1, hg. von Hans-Joachim Hinrichsen, Christian Schaper und Laure Spaltenstein, Schliengen: Edition Argus, 381-461.

Eco, Umberto (1972), Einführung in die Semiotik, München: Fink.

Ehrlich, Robert (Hg.) (2015), Für György Kurtág: „...denn inniger ist, achtsamer auch...", Berlin: Hochschule für Musik Hanns Eisler.

Fischer-Lichte, Erika (2012), Performativität. Eine Einführung, Bielefeld: transcript.

Frigyesi, Judit (2002), „György Kurtág, sSamuel Beckett: What is the Word`, op. 30B (1990/1991)«, Studia Musicologica Academiae Scientiarium Hungaricae 43/3, 397409.

Gadamer, Hans-Georg (1960), Wahrheit und Methode: Grundzüge einer philosophischen Hermeneutik, Tübingen: Mohr.

Genette, Gérard (1993), Palimpseste. Die Literatur auf zweiter Stufe, Frankfurt a.M.: Suhrkamp.

Hartmann, Frank (2000), Medienphilosophie, Stuttgart: UTB.

Hohmaier, Simone (1997), »Meine Muttersprache ist Bartók...«: Einfluß und Material in György Kurtágs ,Quartetto per archic op. 1 (1959), Saarbrücken: Pfau.

- (2001), »Analysis - Play - Composition: Remarks on the Creative Process of György Kurtág“, Contemporary Music Review 20/2-3, 39-50.

(2002), »Mutual Roots of Musical Thinking: György Kurtág, Péter Eötvös and Their Relation to Erno' Lendvai's Theories", Studia Musicologica Academiae Scientiarium Hungaricae 43/3, 223-234.

Hove, Mieke van (2007), „...auf eine sehr komplexe Weise einfach...": Die Bedeutung der Játékok für das CEuvre und den Personalstil von György Kurtág, Berlin: Mensch und Buch. 
Kele, Judit (Rg.) (1996), L'homme allumette / Der Streichholzmann: Kurtág-Portrait, ZDF/ Arte.

Krämer, Sybille (2008), Medium, Bote, Übertragung: Kleine Metaphysik der Medialität, Frankfurt a.M.: Suhrkamp.

Kunkel, Michael (2001), »)...folly for t[w]o...ı: Samuel Becketts «What is the Word György Kurtágs ımi is a szó` Op. 30«, Contemporary Music Review 20/2-3, 51-70.

__ (2008) »... dire cela sans savoir quoi ....: Samuel Beckett in der Musik von György Kurtág und Heinz Holliger, Saarbrücken: Pfau.

Kurtág, György (1979), Játékok zongorára: Spiele für Klavier / Plays \& Games for Piano, Budapest: Editio Musica Budapest.

- (1996), Einige Sätze aus den Sudelbüchern Georg Christoph Lichtenbergs op. 37, Budapest: Editio Musica Budapest.

- (1997), „Nicht potent sein, ohne verliebt zu sein: Meine Begegnung mit György Ligeti«, MusikTexte 72, $28 \mathrm{f}$.

- (2010), Drei Gespräche mit Bálint András Varga und Ligeti Hommagen, hg. von Bálint András Varga, Hofheim: Wolke.

_ (2016), Kocsis Zoli hangjegyfüzete / Zoli Kocsis's Manuscript Book, Budapest: Editio Musica Budapest.

Kurtág, György / Ulrich Dibelius (1997), »`Meine Gefängniszelle - meine Festung`: Porträt György Kurtág, entwickelt im Gespräch mit Ulrich Dibelius«, MusikTexte 72, 29-35.

Neuwirth, Gösta (2011), »Stockende rede - klagendes Lied. Über György Kurtágs `Panaszos Nótaı aus `Signs, Games and Messages«", Neue Zeitschrift für Musik 172/2, 22-27.

Schechner, Richard (2002), Performance Studies: An Introduction, New York: Routledge.

Schulte, Christian (2005), „Cross-Mapping: Aspekte des Komischen«, in: Der Maulwurf kennt kein System: Beiträge zur gemeinsamen Philosophie von Oskar Negt und Alexander Kluge, hg. von Christian Schulte und Rainer Stollmann, Bielefeld: transcript, 219-230.

Spangenmacher, Friedrich (Hg.) (1986), György Kurtág, Bonn: Boosey \& Hawkes.

Wellmer, Albrecht (2009), Versuch über Musik und Sprache, München: Hanser.

Wilheim, András (1997), "Satzfolge und Großform: Der Begriff des ıoffenen Werkes` in den Kompositionen von György Kurtág«, MusikTexte 72, 35-39.

Williams, Alan E. (2001), „Kurtág, Modernity, Modernisms«, Contemporary Music Review 20/2-3, 51-69.

Winkelmann, Helena (2015), „Gespräch in Basel«, in: Für György Kurtág: »...denn inniger ist, achtsamer auch...", hg. von Robert Ehrlich, Berlin: Hochschule für Musik Hanns Eisler, 22-27.

Zenck, Martin (2002), »Beckett after Kurtág: Towards a Theory of Theatricality of a Non-theatrical Music«, Studia Musicologica Academiae Scientiarium Hungaricae 42/3, 411-420. 


\section{Diskographie}

Hommage à Andrei Tarkovsky, Ildikó Monyók, Arnold Schoenberg Chor, Ensemble Anton Webern, Claudio Abbado, Deutsche Grammophon 437840, 1996.

György Kurtág Portrait Salzburg 1993, Ildikó Monyók, Tomkins Vokalensemble, Budapest Festival Orchestra, Péter Eötvös, Col Legno 31870, 1994.

Salzburg Biennale. Festival for New Music 2009, Piroska Molnár, Vokalensemble Zürich, Ensemble Contrechamps, Beat Furrer, Neos 10947, 2010. 